\title{
A review: A Comprehensive Review of Soft and Rigid Wearable Rehabilitation and Assistive Devices with a Focus on the Shoulder Joint
}

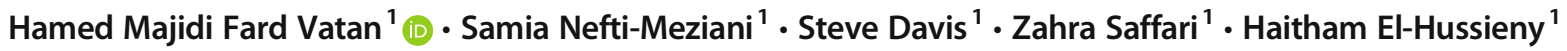

Received: 19 October 2020 / Accepted: 22 February 2021 / Published online: 16 April 2021

(C) The Author(s) 2021

\begin{abstract}
The importance of the human upper limb role in performing daily life and personal activities is significant. Improper functioning of this organ due to neurological disorders or surgeries can greatly affect the daily activities performed by patients. This paper aims to comprehensively review soft and rigid wearable robotic devices provided for rehabilitation and assistance focusing on the shoulder joint. In the last two decades, many devices have been proposed in this regard, however, there have been a few groups whose devices have had effective therapeutic capability with acceptable clinical evidence. Also, there were not many portable, lightweight and user-friendly devices. Therefore, this comprehensive study could pave the way for achieving optimal future devices, given the growing need for these devices. According to the results, the most commonly used plan was Exoskeleton, the most commonly used actuators were electrical, and most devices were considered to be stationary and rigid. By doing these studies, the advantages and disadvantages of each method are also presented. The presented devices each have a new idea and attitude in a specific field to solve the problems of movement disorders and rehabilitation, which were in the form of prototypes, initial clinical studies and sometimes comprehensive clinical and commercial studies. These plans need more comprehensive clinical trials to become a complete and efficient plan. This article could be used by researchers to identify and evaluate the important features and strengths and weaknesses of the plans to lead to the presentation of more optimal plans in the future.
\end{abstract}

Keywords Soft and rigid robotics $\cdot$ Rehabilitation $\cdot$ Assistance $\cdot$ Upper limb $\cdot$ Shoulder joint

\section{Introduction}

One of the most important requirements of every human being is a proper function of his upper limb. This is very important and necessary for any person to perform Activities of Daily Living (ADLs). Upper limb movement disorders, limit the independence of sufferers and greatly reduce the quality of life. "Limiting the ability to perform ADLs eventually leads to an increased risk of mortality and decreased life expectancy of up to 10 years" [1]. Stroke is one of the most common diseases that

Hamed Majidi Fard Vatan

h.majidifardvatan@edu.salford.ac.uk;

h.majidifardvatan@gmail.com

1 Autonomous Systems and advanced Robotics Research Centre, The University of Salford, Manchester, UK are effective in causing this type of disorder in the human body. A stroke occurs when blood supply to a part of the brain is cut off [2]. It is one of the top ten diseases in the world which is ranked second among the known causes of human death in the world [3]. However, according to statistics released by the World Health Organization (WHO), more than 5 million people in the world die annually due to this disease [4].

Upper limb movement impairment in stroke survivors can simultaneously affect the shoulder, elbow, wrist and hand [5]. A significant portion of the world's elderly population is affected by stroke and leaves many survivors with varying degrees and forms of disability [6]. Upper limb defects, for example, in the terms of complete or partial loss of limb function, are also very common in the elderly people [7]. Also, due to the increase in life expectancy in the world and the increase in the elderly community in the future, the number of these patients will increase, consequently and the need to help them will also increase. Other factors influencing movement disorders include some diseases such as cerebral palsy, spinal cord 
injury, Guillain-Barre syndrome, Parkinson's disease, Traumatic brain injury, essential tremor and Multiple Sclerosis $[8,9]$. To address these problems, extensive research studies have been conducted in this field more than two decades ago, specifically aimed at physically rehabilitating patients and helping them perform basic daily activities. One of the effective factors is intensive and repetitive treatments that significantly increase patients' motor skills [7]. The presence of a sufficient number of trained therapists, the availability of rehabilitation devices, financing, having a suitable space to perform movements and considering sufficient time for a treatment are among the effective parameters in the treatment process. For the activities to be effective, the start time, taskorientation, duration of the activities and repetitive task practice (RTP) [10] of training should also be considered [8]. Some motor skills lost due to stroke can be relearned in the rehabilitation process. It is believed that the brain due to its neuroplasticity, i.e. the ability of the brain to reorganize itself through the creation of new neural connections, through rehabilitation devices can repair the damage to itself [6].

Given the limitations mentioned and the potentials presented in the last two decades, it is thought that robotic devices can assist patients in performing two important activities, one is rehabilitation and the other is performing basic ADLs. These devices can perform repetitive tasks on patients with appropriate precision. Also, due to the rapid growth of patient statistics, the long duration of treatment and shortage of the number of skilled therapists or physicians, the use of these robotic devices can be significantly effective in the success of rehabilitation. Robots allow patients to receive the required exercise and also provide tools for measuring patient performance [11]. Generally, robotic devices are divided into two groups such as rigid and soft robots, which researchers have used both methods to advance rehabilitation goals. Of course, a combination of them has been used in some designs, but their frequency has been much less than the two main types. Therefore, the development and optimization process of new devices for the upper limb in the near future can depend on a comprehensive comparison and review of current devices [12].

Review articles related to robots used in rehabilitation robotics field have already been presented by various researchers. Gopura has studied exoskeleton robots and investigated them in terms of mechanical design [13], Bogue has studied the development of exoskeleton and robotic artificial limbs [14], Maciejasz and others, conducted a comprehensive review of the proposed robotic designs to improve the upper limb rehabilitation system [8] and Varghese et al., have investigated wearable robots for upper limb assistance and rehabilitation [15]. Developers of robotic devices have made significant contributions to upper limb rehabilitation by evaluating various technical solutions, and through review articles, this field can be provided for their evaluation. In this review, all the designs presented in the last two decades with a focus on the shoulder joint have been investigated. This is due to the complexity of the shoulder joint, which requires more attention in design and also, it is the first chain in the kinematic chain of the upper limb and its dysfunction can severely limit the functionality of the entire upper limb [1], therefore it can be of great importance. With existing knowledge, a comprehensive portable solution for the whole arm with shoulder joint has not been provided to date and most portable designs have focused on elbow and hand treatment [16].

\section{Search Strategy}

In this review, literature was conducted on Google Scholar, PubMed, Scopus, and IEEE based on relevant keywords. To reach the appropriate articles, filters in the titles of the articles and keywords have been used for this purpose so that the articles close to the target articles can be found. The keywords used are soft and rigid robotics, rehabilitation, assistance, upper limb and shoulder joint, which have been searched in various combinations on the websites. The number of initial articles obtained from all the mentioned sources was 978 . After reviewing them, the more proper articles related to the field of review reached 120 . The most overlap of articles with more keywords used in the first stage is the criterion of this filtering stage. Then articles that did not focus on the shoulder, their presented system was not fully understood and reviewed, and were tasked with moving prostheses instead of real human limbs were excluded from the review. From these articles, 89 articles were selected that had a unique design presented in this field for rehabilitation and basic daily tasks. These articles can be classified into three general groups: Exoskeleton, Exosuit and End-effector robots based on the type of mechanism, whose distribution percentages were $54 \%, 13 \%$ and $33 \%$, respectively. Finally, articles in the field of endeffectors were excluded from this review because they were not wearable, and 60 designs were selected as suitable designs for review. Figure 1 shows the filtering process of the selected articles and Fig. 2 shows the percentage distribution chart of the two final selected designs separately.

Besides, the number of published articles related to the two main structures (Exoskeleton and Exosuit) in the last two decades has been searched on the Scopus website, and the images of their graphs are shown separately in Figs. 3 and 4. As shown in Fig. 3, the trend is slowly increasing from 2000 to 2011, but from 2011 to 2018, statists show a dramatic increase in the production of annual articles in this area, which in 2018 mentioned 180 articles. In 2018, we saw a turning point, and then by 2020 , reported a decreasing trend in this field. Figure 4 also shows that a concept called Exosuit has appeared in the titles and keywords of articles since 2012. Of course, in the years before this date, as shown in Table 1, we have witnessed the use of Exosuit designs since 2004, but with other names such as soft exoskeleton. As shown in Fig. 4, this trend has 


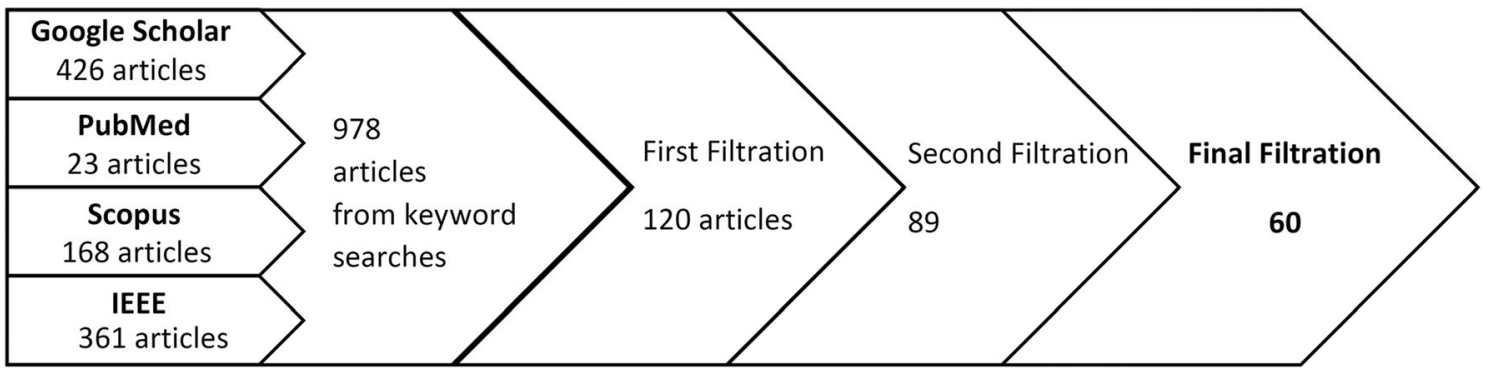

Fig. 1 Filtering process of selected articles

been increasing from 2012 to 2020, from 2 in 2012 to 25 in 2020. Due to the increasing requirement for lightweight and portable systems in the near future, we will see a further increase in the number of articles in the field of Exosuit.

\section{Upper Limb Biomechanics}

As regards that, the main goal is to find better and easier solutions to help subjects with movement disorders, it is very important to know the anatomy and human body biomechanics. Complete and accurate knowledge inspires when designing robotic based systems. Familiarity with the science of neuroscience and biomechanics, which is effective in identifying neuromuscular diseases and rehabilitation, can be effective in the field of Exoskeletons and Exosuits that have cognitive and physical Human-robot interaction (HRI) factor. Due to the fact that the human body is considered as a framework in soft robotic systems, bio-inspiration is considered an important issue when designing $[13,15]$.

\subsection{Parts of Upper Limb}

The upper limb is suspended from the trunk and it is divided into shoulder, elbow, forearm and hand [19]. Unlike the lower limbs, which are used for mobility, support and stability, the upper limbs used for hand placement in very mobile space. Also, anatomically, the upper limb of the human body is mainly divided into three main joints: shoulder, elbow and wrist [20].

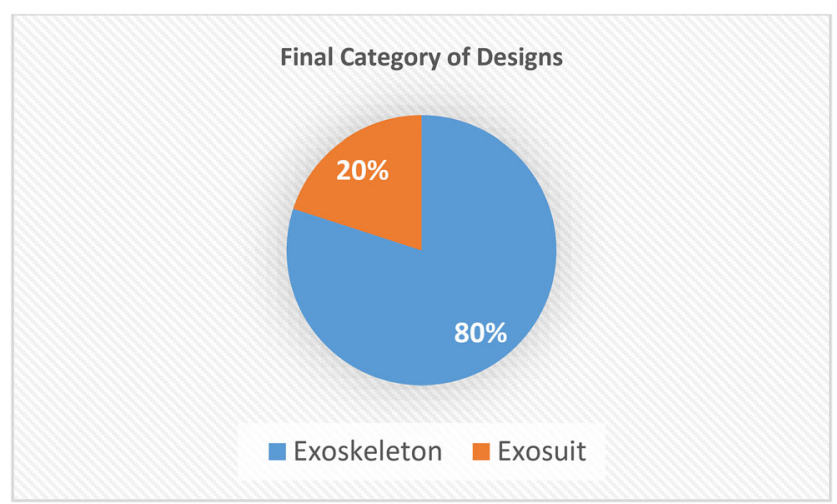

Fig. 2 Distribution percentage chart based on final design classification

\subsubsection{Shoulder Joint}

Three bones called the humerus, clavicle and scapula are the bones of the shoulder and four articulations called scapulothoracic, acromioclavicular, glenohumeral and sternoclavicular belong to the shoulder joint, but glenohumeral is referred the main connection of the shoulder [15]. The sternoclavicular junction is the only interface between the shoulder and the axial skeleton of the body. However, when describing the scapular movement on the thorax, the sternoclavicular is considered an articulation [13].

The glenohumeral joint (shoulder joint) allows the arm to move more freely on three general axes, extending the reach of the hand. The arm movements in this joint are abduction, adduction, flexion, extension, internal rotation, external rotation and circumduction [19].

In the design, the shoulder complex is often modelled as a ball and socket joint, also referred to as a spheroid joint [21], which is formed by the proximal humerus and the glenoid cavity of the scapula. However, the position of glenohumeral joint rotation center changes with the upper arm movements. Important movements of the shoulder complex are flexion/ extension, abduction/adduction, and internal/external rotation, and in the most cases this complex is known in research as a limb with 3 Degrees of freedom (DOF)s [13] and in some other designs internal/external shoulder rotation is used less than other shoulder DOFs [22]. Due to constantly changing of the center of rotation of shoulder joint, it is necessary for some designs to be modelled as a 5 or 6 DOFs system, instead of modelling as a typical ball and socket joint [15]. In general, the shoulder complex movements are divided into 3 movements in the shoulder and other movements occurred in the Shoulder Girdle.

While this assumption is considered almost exclusively for small glenohumeral motor angles and is significantly deviated during larger movements because the thoracohumeral joint has a movable center of rotation [23, 24]. Large misalignments occur in the shoulder through altered motor axes. For example, the correct estimated center of rotation in the shoulder mentioned in [24]. Also, the position of the humerus from 0 to $180^{\circ}$ is drawn in [23] to understand the displacement of the centers. 
Fig. 3 Number of Exoskeleton articles published per year (TITLE-ABS-KEY (exoskeleton) AND TITLE-ABS-KEY (robotic)) [17]

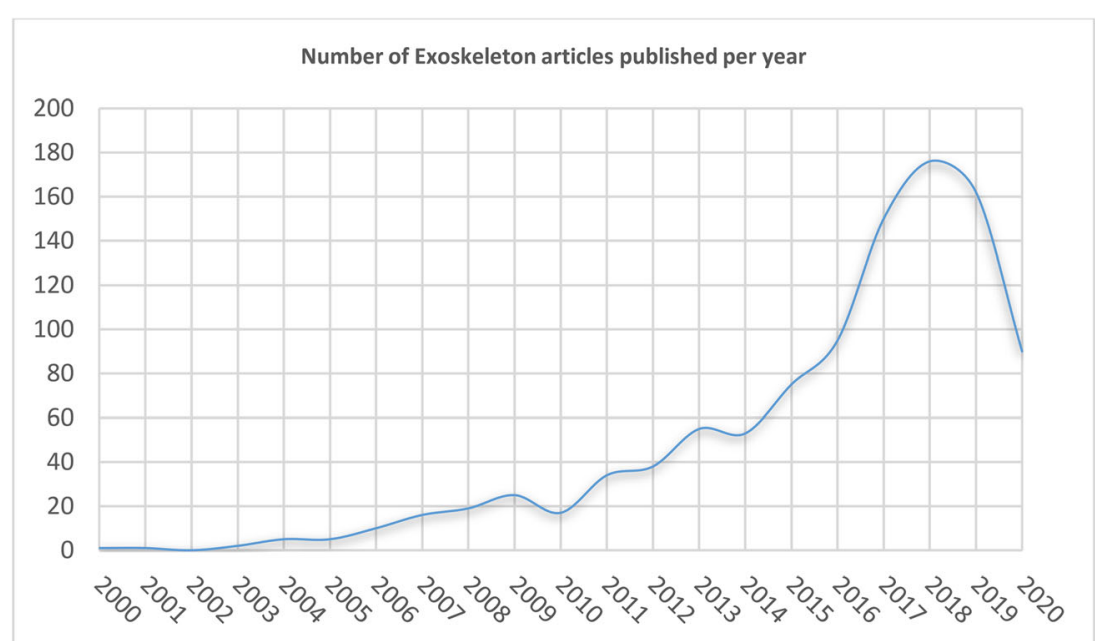

There are several ways to deal with the additional translation movement and it has been studied by different groups [25-27]. One strategy is to add some passive joints. Of course, adding passive joints to the actuated skeleton also destroys the robot's statically determination and this not only gives the patient more freedom but also reduces the mechanical guidance and support of the limb [23]. In exoskeletons, since the human arm is almost fixed in the robot arm, the relative distance between the arm holder and the Center of Rotation (CR) of the human shoulder joint is almost constant. Therefore, the distance between the arm holder and the CR of the robot shoulder joint should be adjusted on average according to the shoulder movement to reduce the effects of the disease caused by the CR position difference between the robot shoulder and human shoulder [28]. Shoulder girdle movement is very important for orienting and stabilizing the arm during daily activities. This movement is a nonlinear movement that is determined by the orientation of the humerus and is of course different for each person. Therefore, it is inappropriate to use this motion before accurate calculation, because if an exoskeleton robot fails to mimic the patient's shoulder girdle movement well, the robot's axes will not match the patient's body, reducing Range of Motion (ROM) and discomfort for patients in the long run [25].

\subsubsection{Elbow and Forearm}

The elbow is made up of three bones, the radius, ulna and humerus, but is primarily modelled as a uniaxial hinge joint $[13,21]$. The size of the elbow joint can be used to find the axis of rotation of the elbow joint in exoskeleton robots, and this is not a problem. The main movements in the elbow joint include the extension and flexion of the forearm. Forearm movement occurs by the ulna and radius bones at the distal end and by rotating the inner bone on the ulna head (Pronation). However, to convert the palm-posterior position to the palm position, the radius must also rotate on the ulna side (Supination) [19]. In general, the elbow and forearm are each a member with one DOF.
Fig. 4 Number of Exosuit articles published per year ((TITLE-ABS-KEY (Exosuit) AND TITLE-ABS-KEY (robotic)) [18]

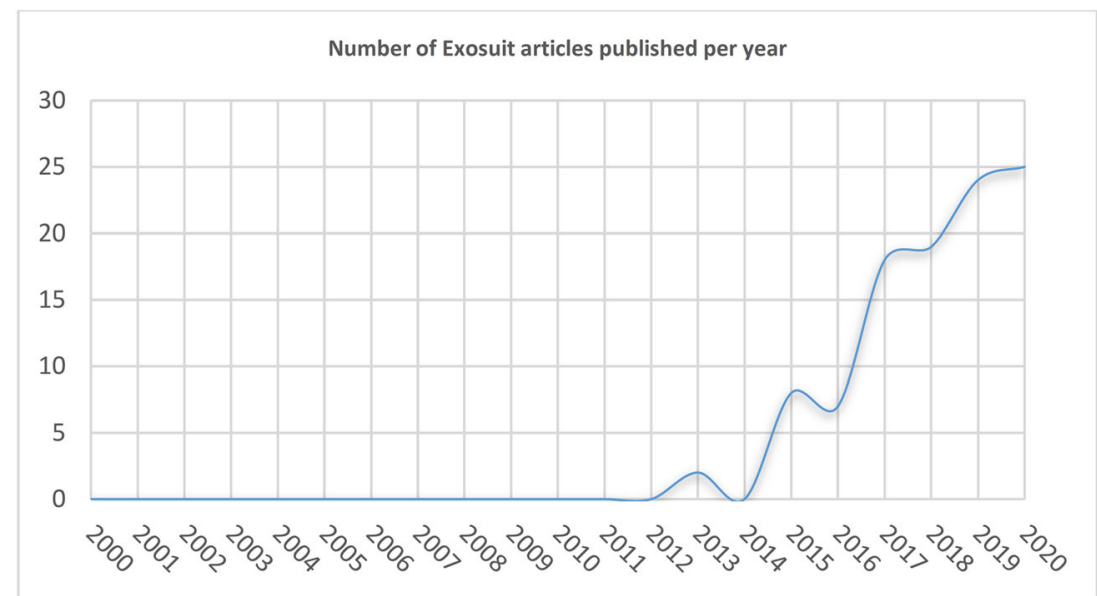




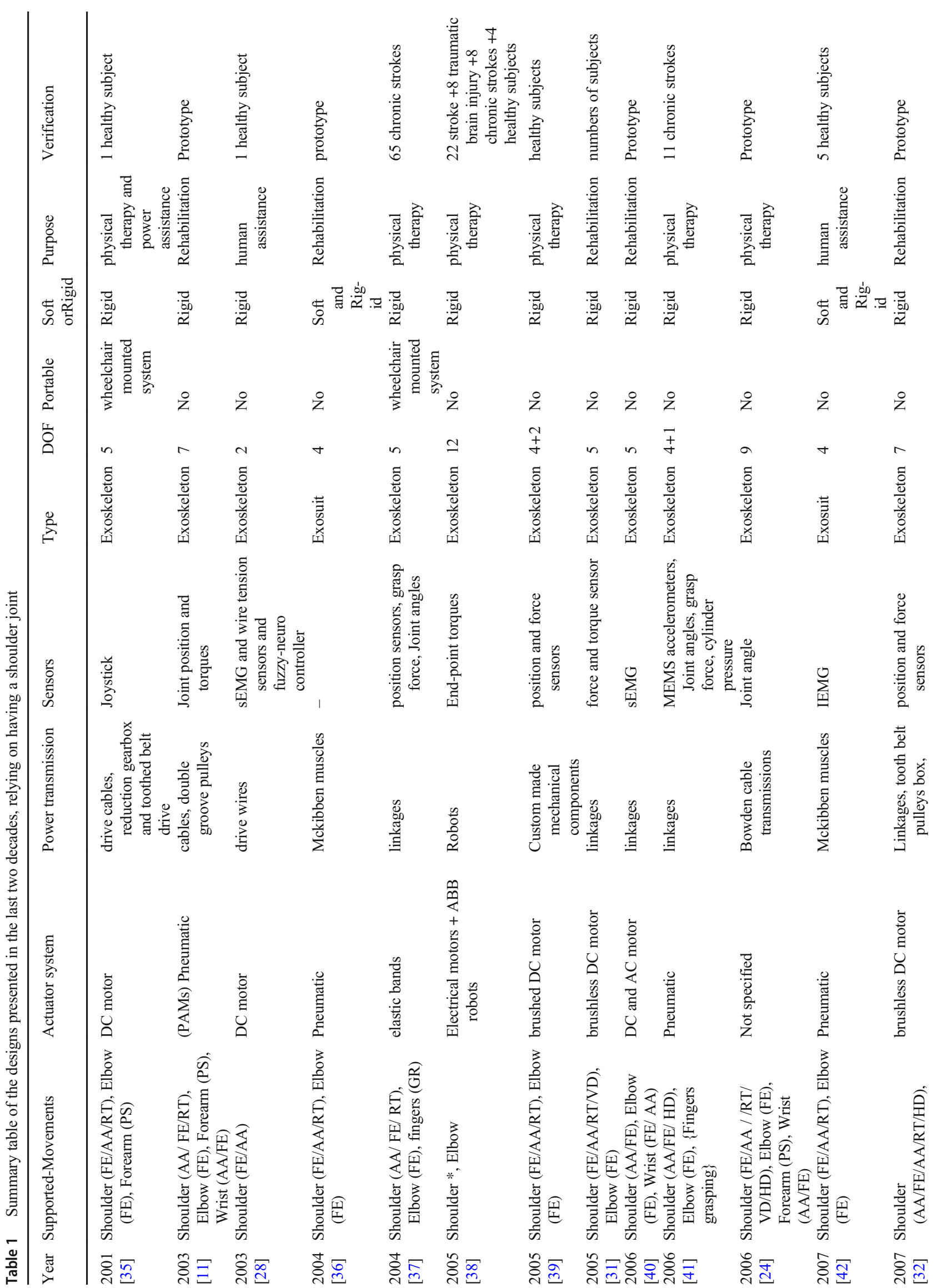




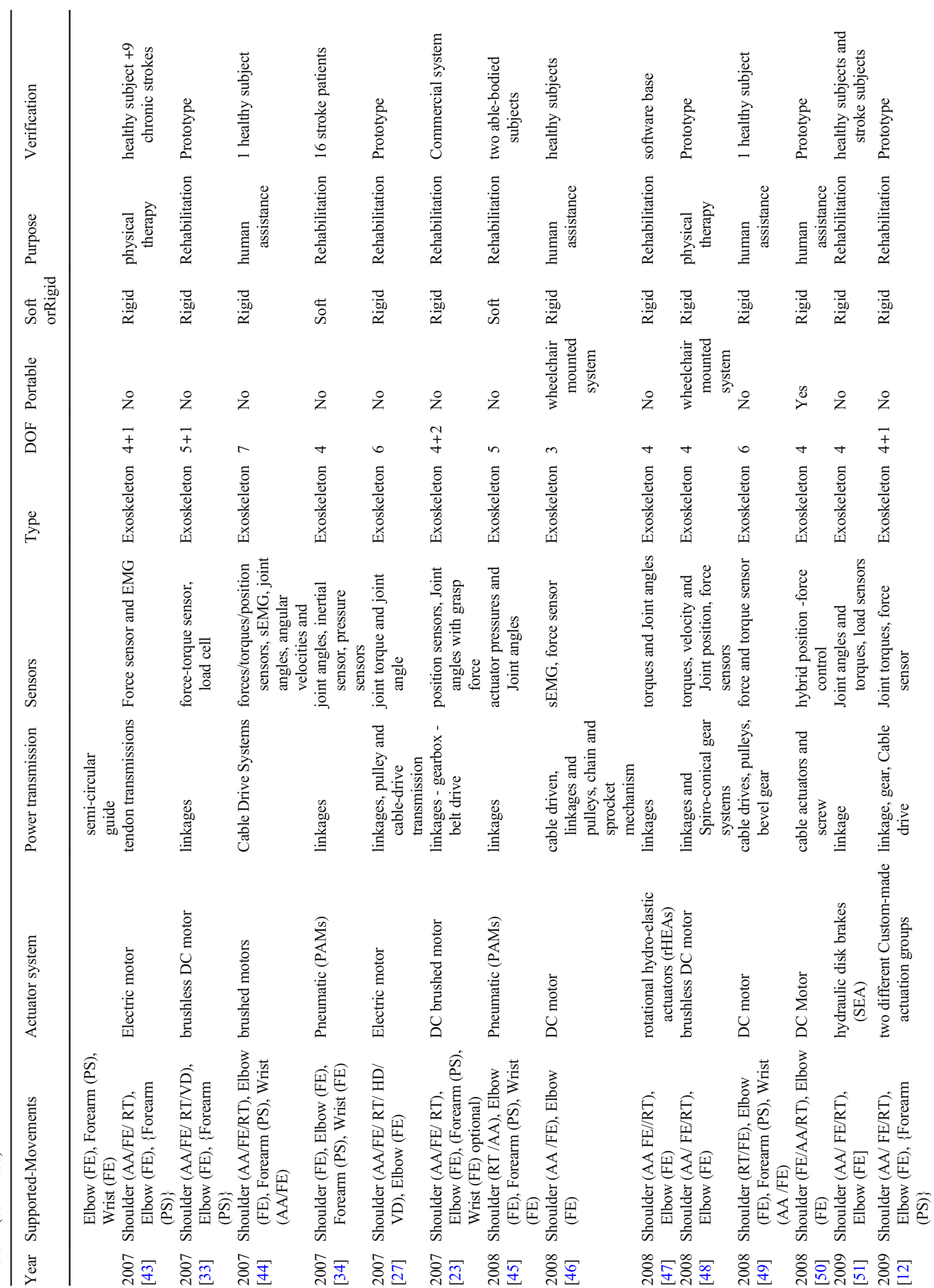




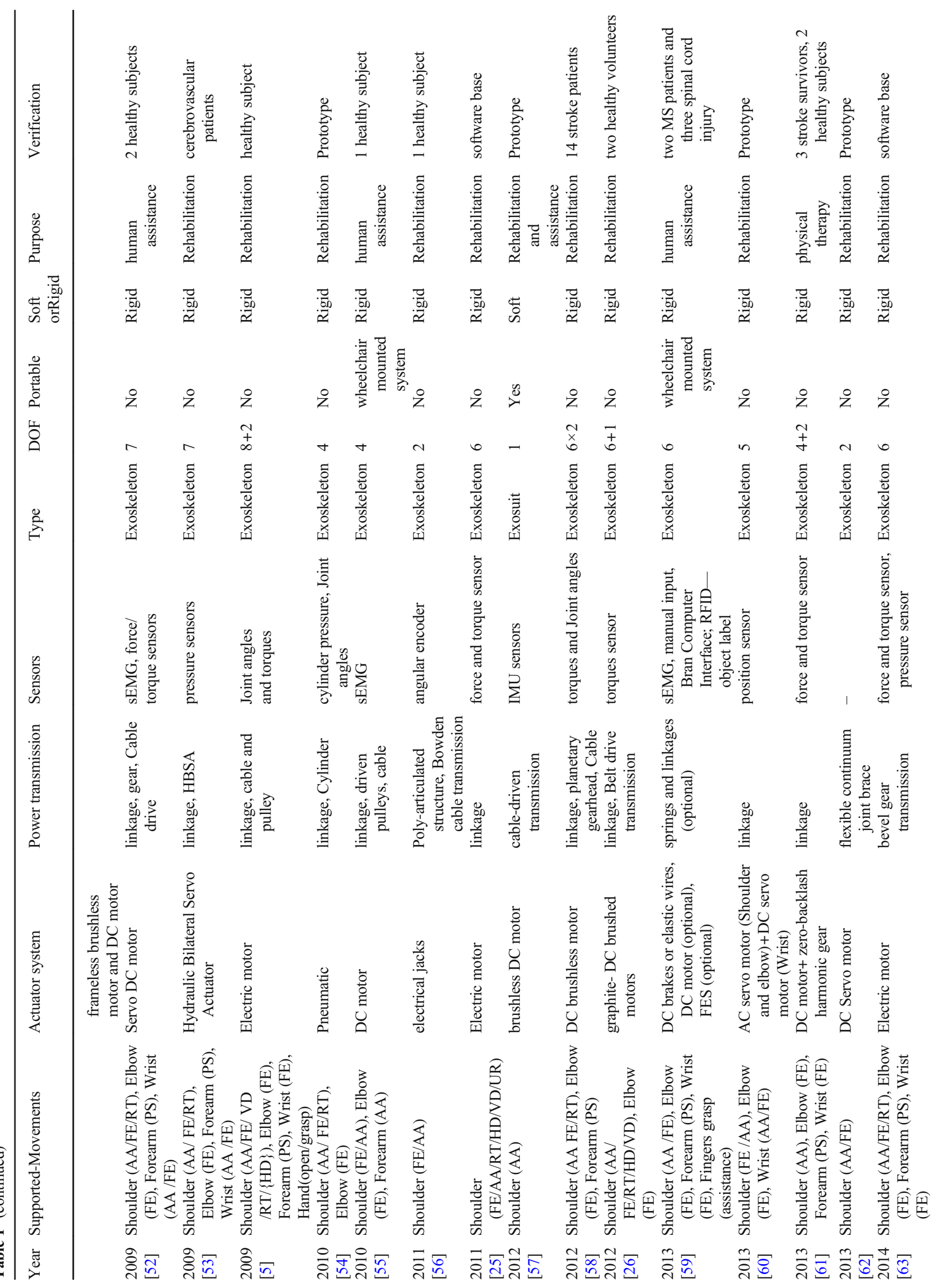




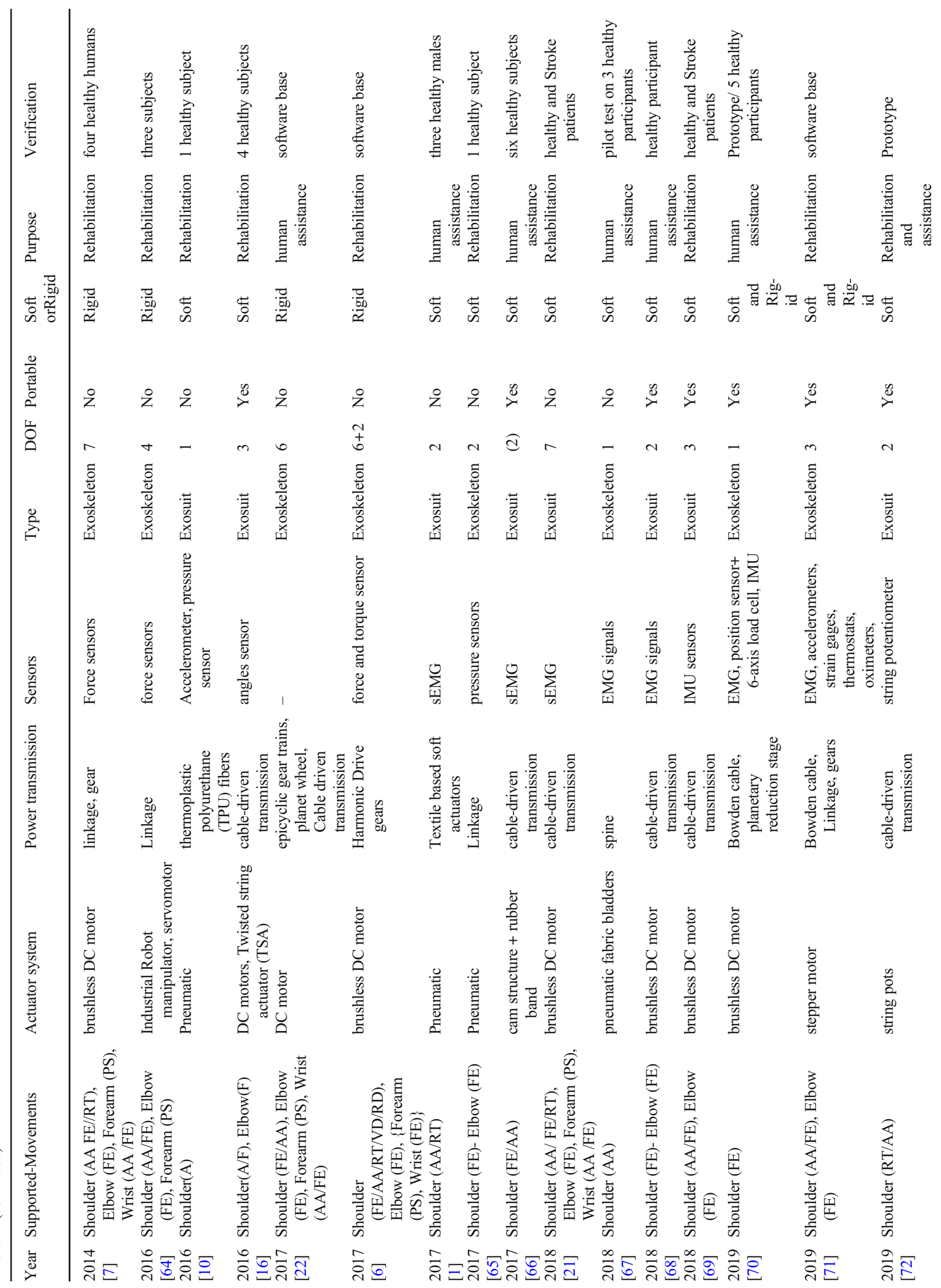




\subsubsection{Wrist}

The wrist joint includes abduction, adduction, flexion, extension, and circumduction movements. These movements, together with the movements of the upper limb joints to be placed in a wide range of positions relative to the body [19]. The carpus joint is a formable joint that connects the forearm to the hand, and in some sources, the wrist joint has been interpreted as an oval joint [21]. In general, the carpus has been introduced as a member with two degrees of freedom in most studies [20, 22, 24]. In other words, when we consider that extension and flexion movements have one axis and also ulna and radius movements have one other axis, there will be a slight offset between these axes, the researchers measured it to be about $5 \mathrm{~mm}$ and it is shown in [13]. Finally, each of the eight carpal joints can create only a limited amount of motion, and the set moves together as an allied unit.

\subsubsection{Hand}

The hand bones are made up of the carpal bones, metacarpals, and phalanges. The five fingers of the hand are the thumb, index, middle, ring and little fingers. The hand is used as a mechanical as well as a sensory tool. One of the most important mechanical functions of the hand is to grip and manipulate objects. The sensory cortex of the brain is also dedicated to the interpretation of hand information, especially from the thumb, which is relatively large compared to many other areas of the skin [19].

The bones of the fingers are phalanges. The thumb has two phalanges, while any other finger has three phalanges. The metacarpophalangeal joints are biaxial condylar joints (ellipsoidal joints) that allow flexion, extension, abduction, adduction and circumduction.

\subsection{Muscles}

\subsubsection{Shoulder Muscles}

Some shoulder muscles, such as the Levator scapulae, trapezius and rhomboids, connect the clavicle and scapula to the trunk. Other muscles connect the clavicle, scapula, and trunk to the proximal end of the humerus. These muscles include the pectoralis minor, pectoralis major, teres major, deltoid and latissimus dorsi [19]. The most important of these muscles are the four rotator cuff muscles (infraspinatus, subscapularis, teres minor and supraspinatus muscles) that connect the scapula to the humerus and support the glenohumeral joint. The shoulder has a total of 6 important muscles: Deltoid, four rotator cuff muscles (Infraspinatus, Subscapularis, Supraspinatus and Teres Minor) and Teres Major [29]. 


\subsubsection{Elbow Muscles}

The most important elbow muscles involved in flexion and extension movements. The Brachialis, Biceps Brachii, Brachioradialis, and Coracobrachialis muscles are involved in elbow flexion and the Triceps brachii and Anconeus are also responsible for elbow extension movements [21].

\subsubsection{Forearm and Wrist Muscles}

The forearm muscles can be discussed in two parts; anterior and posterior. Anterior muscles are formed in four layers from superficial layer to deep layer [30]. Also, the forearm, which has been expressed in research as a limb with a degree of freedom, is of great importance in daily activities such as turning the key to open the door, opening a drinking water bottle, and so on. Movement of the wrist joint can increase the direction of achievement to increase the flexibility of the grip. The flexor carpi radialis muscle plays the most important role inflexion. The extensor carpi ulnaris muscle plays the most important role in extension. Flexor carpi ulnar plays the most important role in adduction and extensor carpi radialis plays the most important role in abduction [21].

\subsubsection{Hand Muscles}

Generally, the hand muscles are divided into five different muscles. The first group is the Dorsal interossei muscles, which are the four muscles attached to the metacarpal bones of the fingers; the function of these muscles is to assist in abduction and adduction movements. The second group are the Palmar interossei muscles, which are the three muscles attached to the metacarpal bones of the fingers. The function of these muscles is to help the pulling movement of little, index and ring fingers in the transverse direction. Next group are the Lumbricals muscles, composed of four small muscles that cause extension and flexion movements. The composition of the muscles of groups 1 to 3 are called metacarpal muscles. The fourth group of muscles is called Hypothenar, which consists of four different muscles and is located on the little finger, and its function is to help flexion and extension of the little finger. The final group of hand muscles is called Thenar, which consists of four different muscles whose task is to help thumb to move in different directions. Also, muscles of the fifth group can make contact between the thumb and all four other fingers of the hand [29].

\subsection{Range of Motion}

Upper limb movements are generally divided for two areas, one for performing important daily activities and the other for performing all tasks. In most cases, researchers have provided plans that can be used to help perform important daily tasks. Researchers have introduced ROM of different parts of the human upper limb in various studies and then compared the data obtained from the design of the systems provided by them with the original data and through this study, the percentage of motion overlap of the proposed systems with the actually required amplitude has been measured [11, 31-33]. For example, Sugar et al., considered a sample with a specific height and weight as an index to obtain their data and carried out the whole design accordingly. Finally, anthropomorphic data can be converted by scaling a first model based on the weight and height of the new user for other cases [34]. ADLs include tasks such as drinking, eating, combing hair, etc. The complete mechanism should be able to move the shoulder with 3 DOFs, the elbow with 1 DOF, the forearm with $1 \mathrm{DOF}$, the wrist with $2 \mathrm{DOF}$, and also include the action of gripping in the fingers [23]. For example, Carignan et al., compared the upper limb movement range with 7 different robot designs using an average data of 39 men for the range of motion of the human arm. Also compares 5 robot designs with the average data obtained from the bodies of 39 men in relation to the maximum torque applied to the limbs [31].

\section{The Framework of the Literature}

As stated in the second part, 60 designs were ultimately selected as the final design for review, which the following frameworks were considered for comparing the designs:

1. Types of mechanism

2. Rigid or soft robotics

3. Portability

4. Types of actuators

5. Types of sensors

6. Types of power transmission systems

7. Types of control units

8. Status and details of clinical tests

In the following, additional explanations will be provided for each of these sections. The results of reviewing all designs are presented as a summary table in Table 1, and for working groups that have had different designs, each of their designs is listed in the table.

\subsection{Types of Mechanism}

As mentioned in Section 2, a total of three types of mechanisms were found in the literature evaluation that was used in the field of rehabilitation and performing the main ADLs, among which end-effectors were removed because they were not wearable. Easy adjustment with different arm lengths is 
one of the most important advantages of end-effector-based robots. Their disadvantage is also that in general the arm posture is not completely determined by the robot and have interacted from one point. As a result, their ROMs are limited, and exoskeleton robots are generally better suited for training activities that require a large ROM [23]. The exoskeleton is an external mechanism that transmits the torques and forces generated by actuators near human joints through the joints they make with the outer part of the upper human limb [44] and Exosuits are soft exoskeleton that the anatomical structure of the human body forms its main framework [74]. The images of all three samples are shown in $[20,73,75]$, and in this paper, as mentioned earlier, only two designs, Exoskeleton and Exosuit, have been examined for their wear ability.

\subsubsection{Exoskeleton}

Exoskeletons are based on the architecture of industrial robots and include actuators, mechanisms and similar materials. They also include the lower and upper limbs, which act directly on the human body [76], however, this paper investigated only the upper limbs. Despite the existing complexities, many upper exoskeletons of rehabilitation have been developed and tested in the last two decades [70]. Ideal robotic rehabilitation devices should be able to: 1) train the full workspace of the human body, 2) activate the joint to stimulate precise ergonomic movements in the patient, 3 ) should not cause discomfort or safety hazards to be used when moving. According to current research and knowledge, there is no wearable or endeffector based rehabilitation device that has all these benefits and become a complete system [24].

Rehabilitation exoskeletons have improved the quality of life of patients with neuromuscular diseases such as stroke or spinal cord injury. Also, in the case of using exoskeletons, this system will delay the onset of fatigue by reducing muscle activation in healthy users when doing physical work with the upper limb, while users with mobility impairments will be able to move their upper arm through the exoskeleton [67]. As mentioned in the second part, 48 out of the 60 existing designs included exoskeletons, in which $73 \%$ of them are provided as stationary, $17 \%$ portable and $10 \%$ as a wheelchair mounted system. Also, $72 \%$ of the designs are presented as rigid, $23 \%$ as soft and $5 \%$ as a combination of both soft and rigid designs. In [7, 11, 39] some images of these designs was shown.

Exoskeletons can have different DOFs depending on the design and expected performance [77]. In the proposed designs, the exoskeleton with one DOF $[67,70]$ to designs with 12 DOFs are provided [58]. In general, the human arm movement in exoskeletons is usually designed with 7 DOF [31]. As the number of DOFs increases, the complexity of the system increases, although, in the case of whole-body rehabilitation systems, the number of DOFs reaches nine, ten or more $[5$, 38].

As mentioned, exoskeletons are similar to the devices introduced in [6, 20, 78] and [31] wearable biomechanical systems that are installed parallel to the subject limb, expand either in the entire upper limb or in certain parts of it. In the exoskeleton, the axes of rotation of the robot must match the axes of the anatomical rotation of the patient because having a mismatch between the exoskeleton and the limbs can have devastating effects on the rehabilitation process or on long-term use of these devices [24, 57]. Among these, devices have been provided that have the ability to self-align $[56,79]$. These devices are portable and stationary and are provided with a variety of actuators and sensors, the details of which are presented in Table 1. For example, Bogue has presented different examples of exoskeleton devices [14]. The complexity of the mechanical algorithm and control of such devices is usually significantly higher than end effector devices, which of course, the complexity of these devices also increases with increasing the number of DOF [8]. The center of glenohumeral joint ( $\mathrm{CGH}$ ) changes according to the different directions of the humerus, which is caused by the shoulder girdle movements. Therefore, the shoulder girdle movement must be considered in the kinematics of the robot shoulder mechanism. Regardless of this, the mismatch between the rotation axis of the patient's shoulder and the robot shoulder not only creates a limited workspace for rehabilitation but also causes discomfort to patients $[25,52]$. Some researchers have also suggested the addition of passive joints as a way to prevent the adverse effects of misalignment on the joint [70], which has been and will be fully explained.

Exoskeleton devices have a mechanical structure that reflects the skeletal structure of the patient's limb. The use of an exoskeleton-based approach allows the patient to control independently and simultaneously the specific movement of the arm in many joints. However, to prevent injury to the patient, it is necessary to adjust to the length of the patient's arm [8, 32]. A significant disadvantage of current robotic devices is that they cannot properly match the movement of the upper human limb [27]. Rigid exoskeletons have rigid mechanical bodies $[23,35,80]$ and this capability allows them to transmit forces and torques without the anatomical equivalent (user limb) and experience different load ranges. This parameter also makes it possible to use a simpler control system and also to perform more complex displacement movements. The mentioned advantages make it possible to use large forces and torques for such systems, which are often used in the military and industry. These systems can also be used in rehabilitation for patients who have less spasm in their joints or need more force and torque [15]. Some of the disadvantages of these systems include poor dynamic response speed, interference with joint movements that cause the wearer to deviate from 
normal movement patterns, limitation of wearer flexibility, increased system metabolism, large inertia regulation mechanism, and poor pairing between humans and machines which causes low energy efficiency and deviation from normal human movement [21]. We can also point out to their high weight, which this requires supplying more force and torque to move and ultimately the need to provide greater sources of power [80].

Most of the design's body is made of aluminum [11] because aluminum is a low-density material with suitable strength properties. Carbon fiber is also an ideal candidate for exoskeleton's body material. Recent advances in manufacturing techniques such as $3 \mathrm{D}$ printing of carbon fiber-reinforced structures make it possible to achieve complex geometries. One of the advantages of these methods is that they make a combination of plastic, aluminum and reinforced steel with carbon fiber [6, 43].

There are different mechanisms for ensuring the safety of design systems [8]. One solution is to place mechanical and electrical stoppers to limit the ROM in the human body. In one design, researchers limited speed and torque to prevent sudden hand movements by control programs [13]. Also, the mechanical design should be done in such a way as to improve inertia reduction [33]. Therefore, the challenge in designing the exoskeleton is to reach a conceptual agreement among power, workspace, dynamics and weight [81]. Also, for the robot to function properly, it must have low friction, low inertia and a backlash-free system [39]. Although industrial robots are highly resistant to the upper human limb and should not be in physical contact with patients, in some cases they have been used to reduce costs [58]. Therefore, having a low intrinsic impedance of designed systems is one of the important factors in designing rehabilitation systems for upper extremities [8]. Most haptic devices use a basic form of impedance control in which Cartesian forces in the category using Jacobian fall in the commands of common torques. The most important advantage of this method is that it does not require the calculation of inverse kinematics and is stable at low impedances. Also, in teleoperation, the exoskeleton aims to generate contact forces in the exoskeleton category, which are the reproduction of forces felt by the slave arm. While in virtual reality programs, a virtual environment is used instead of a slave arm to generate force commands [33].

\subsubsection{Exosuit}

The systems presented in articles $[16,36,57,59,72]$ are known as Exosuit devices. Unlike the rigid systems used in Exoskeleton, Exosuit uses the anatomical structures of the body to shape the robot frame [74]. In other words, the most important difference between Exoskeleton and Exosuit is the latter's soft texture, which includes a fabric base frame that has the ability to transfer flexibly.
These systems are made of appropriate clothing in appearance and are lighter and more portable than Exoskeletons. They also use the structural integrity of the human body to transfer forces between different parts of the body [68]. Due to the lack of a rigid skeleton, the user's natural movements in Exosuit are not limited [73]. Exosuit exerts a force on the joints in parallel with the muscles, Which can improve the effect of the auxiliary force and the connection of the device system [21]. The use of Exosuit systems due to their lightweight makes performing movements and applying forces and torques require less initial energy, which in turn increases the time of using the intended energy source compared to rigid exoskeletons in the same time interval. Due to its compatibility with the user's body and its lightness, it makes it possible to cause less movement and misalignment injuries than rigid devices. Therefore, the inherent adaptation of Exosuit devices to the human body facilitates their mechanical design [15]. It is also possible that due to their design, they can be hidden under people's clothes in the near future, which can have very positive effects on patients in terms of social psychology [57, 68, 72]. According to the 12 designs reviewed in this article, $58 \%$ of the designs are portable and $42 \%$ are stationary. Also, $83 \%$ of them are presented as soft and $17 \%$ as a combination of soft and rigid.

One of the advantages of Exosuit systems is the materials used in their body design, which are much cheaper than Exoskeleton systems, and elastomers and fabrics are mainly used to make them $[1,57,72]$. An important result of using cheap materials for these devices is their lower cost and portability, which allows them to be used by a wider range of patients. Also, due to this feature, their application at patients' home has become more possible and they can have industrial applications as well [68]. Disadvantages of these devices include the lack of a rigid frame to transmit forces and torque. The important challenge here is that all the forces and torques will be transmitted through the patient's body, and due to the lack of a fixed and rigid frame, some problems will be raised. In this case, it is not possible to connect the actuators and sensors directly to the mainframe, and in principle, they must be transmitted to the limbs through secondary systems and power transmission mechanisms. Also, their control systems are complicated due to the use of user biomechanics and are one of the challenges of these systems [15]. In some Exosuit devices, for example, in addition to generating a natural force to move the limb, a shear force is also generated, which should be minimized because it has no effect on the rotation of the limb and only rubs the device on the skin which can be painful [66]. However, putting appropriate distance between the transmission system connections and the body parts can significantly reduce the shear forces when stimulating the actuator on the Exosuit trunk [16].

When dealing with system modelling, the dependence of the model parameters on the arm complexion of the wearer is important. In addition, the flexibility of the Exosuit makes it impossible to be placed on the arm always in the exact 
position [73]. Given the above, the expectations we should have from an Exosuit system can be as follows [57]:

1. easy to wear and undress

2. as light as possible

3. cost-effective system

4. create forces that help them during the rehabilitation process and measure the position of the arm

5. compatible to improve safety and no rigid elements should be used

6. compatible with anatomical changes and possible misalignments

Table 2 introduces some of the commercialized samples. Most of the commercialized systems have been provided as shoulder-centric to help healthy people in industrial environments, and according to the existing knowledge and studies, a portable shoulder-centric commercial system has not been provided so far.

\subsection{Rigid and Soft Robotics}

Rigid robots are older than soft robots. Rigid robots have been used frequently in military systems, industry and etc., but soft robots have been growing in recent years due to the limitations of rigid robots, such as high weight, low portability, etc. In the reviewed articles, three general designs have been used, i.e. rigid $[11,78]$, soft $[34,66]$ and a combination of rigid and soft robots [45, 70]. In general, the limitations of rigid robots mentioned in the previous sections have led to the emergence of soft robots. In the studied designs, the percentage distribution diagram of devices is as shown in Fig. 5:

REHAROB [38], ARMin III [23], CABexo [22] and CLEVER [6] are examples of systems provided for rigid robots and RUPERT IV [45] and ExoFlex [73] are examples of systems proposed for soft robots.

\subsection{Portability}

Portability parameter for the patient is important because these devices often help patients perform basic ADLs and help them

Table 2 Comparison table of Exosuit commercialized samples

\begin{tabular}{llll}
\hline Item & Company name & Product name & Area of help \\
\hline 1 & SUITX & ShoulderX [82] & Shoulder \\
2 & Ekso Bionics & EksoWork [83] & Shoulder \\
3 & Myomo & Myomo [84] & Elbow and Hand \\
4 & Ottobock & Paexo Shoulder [85] & Shoulder \\
5 & Ekso Bionics & ExoUE [86] & shoulder and elbow \\
\hline
\end{tabular}

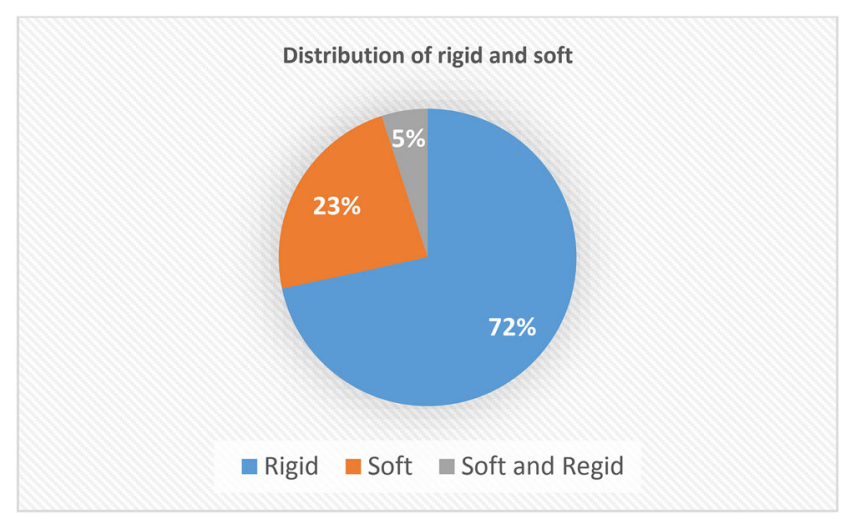

Fig. 5 Percentage distribution diagram of designed rigid and soft devices

in performing more rehabilitation activities at home without the presence of a doctor or technician. There is a total of three types of capabilities in robots designed for rehabilitation, including portable $[36,57,50,68]$, stationary $[1,11,43,60]$ or wheelchair-mounted systems $[35,55,59]$. Figure 6 shows the distribution chart of these designs. When designing portable skeletons, the classic tradeoff between power and weight always emerges [31], therefore the weight of the wearable robot is a very important factor in its portability [33].

\subsection{Types of Actuator}

Robots can be classified according to the types of actuator used in the designs. The types of actuator used in the system are derived from the choice of energy source [8]. In general, three types of the actuator are used for rehabilitation robots, which are electric [7], pneumatic [45] and hydraulic [51]. Of course, some of the designs are not included in this general classification, then we classified them into a separate group called others. The location of the actuators is an important factor, especially in exoskeleton-based mechanical structures, where the actuators are located near the connection on which they operate. Figure 7 shows their distribution graph, which, as it turns out, most of the actuators used in the designs are electrically, and a small percentage of them have used other types.

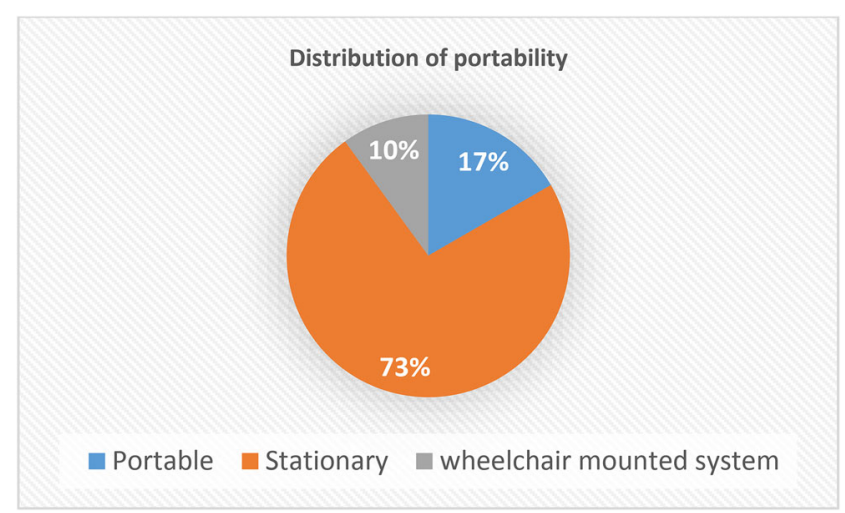

Fig. 6 Distribution graph of portability of designs 


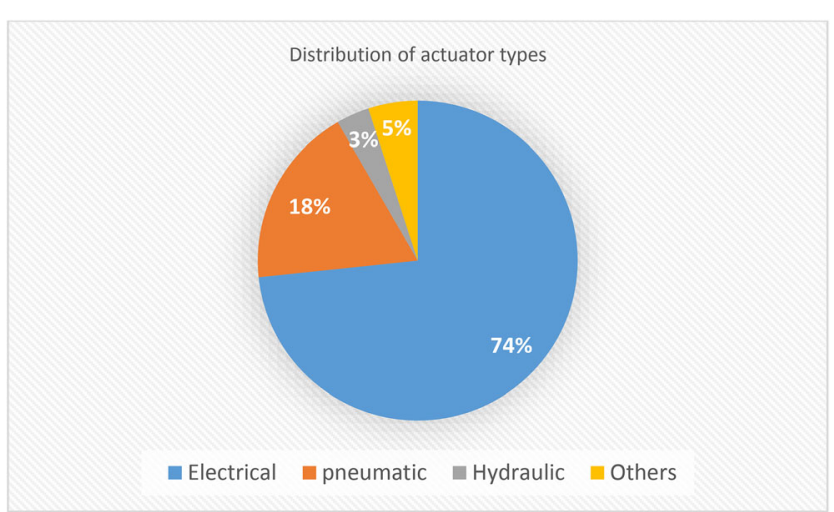

Fig. 7 Distribution chart based on actuator type

\subsubsection{Electrical Actuator}

As mentioned, more than $70 \%$ of the actuators used in the designs were electric actuators. These actuators often include DC and AC motors, although more DC motors are used. The possibility of storing energy in batteries and their ease of use in DC motors is one of the reasons for using DC instead of AC motors in robotic systems. In other words, DC motors are used in portable robots that need smaller forces and torques, and $\mathrm{AC}$ motors are used in stationary industrial robots that need to provide larger forces and torques. In practice, DC motors outperform than $\mathrm{AC}$ motors for an equal amount of energy entering the system. Most upper limb rehabilitation robots are activated by electric drives [87]. One of the reasons for preferring electric actuators over other actuators is the compactness of electric motors and the ease of control of these systems [35]. Also, the system consists of an electric battery and motor and it is lighter and smaller than a pneumatic system with the same specifications, therefore more suitable for fully portable and wearable auxiliary systems. Galiana et al., "have shown that the energy density, i.e. mass in each energy stored of a lithium battery is larger than the compressed air system, and mechanical coupling is placed at the end of the actuator to secure the system and ensure that the motor receives no axial force or off-axis torque that causes it to malfunction" [57]. In terms of system safety, whenever an abnormal event is detected, the safety circuit immediately reduces the power of the motor drives. For example, Nef et al. equipped their system with a passive weight compensation system and showed that the robot does not fall after losing power [39]. If the drives are back-drivable, the robot can easily be moved manually by a therapist to relieve the patient of an uncomfortable posture [23]. For example, Pang et al. has developed a new system for performing internal and external rotation movements of the shoulder joint by means of a curved rail, a gear system, an engine and gearbox [20]. Kim et al. also presented a system with electric actuators and a gear and pulley transmission system [68].

\subsubsection{Pneumatic Actuator}

Few systems use pneumatic actuators. Pneumatic actuators are lighter and have lower intrinsic impedance, and also due to the need for pneumatic pressure to start, most of these systems are used in a stationary and limited area [41] or a small compressor is mounted on the patient's wheelchair [8]. These actuators are presented in two different designs in the form of pneumatic cylinders [54] as well as McKibben actuators [45]. Pneumatic cylinders that are embedded in different parts of the upper limb with different systems and do the desired operation based on the one-way or two-way cylinders and compressed air force according to the design [41]. McKibben actuators were also developed for prosthesis research in the 1950s and 1960s [36], the structure of that is shown in [42]. These types of actuators, which also have a very good power/weight ratio, meet the need for safety, simplicity and lightness [11].

Pneumatic Artificial Muscle (PAM) is also derived from the design of McKibben actuators, which, when the bladder is subjected to compressed air, the diameter of its actuator increases, and as its volume shortens, stress is created at its end. In other words, they are a special type of pneumatic actuator with an internal bladder surrounded by a braided shell with flexible but non-expandable threads. Due to their special design, this actuator is shortened like a contractile muscle under pressure. The advantages of these designs include natural adaptability, low mass, inherent safety, high power to weight ratio, low cost, and ease of construction [11]. Due to the relatively low energy density due to the compressed air tank, these systems cannot operate as a fully mobile wearable system, which is one of the disadvantages of these systems [57]. It is very important to note that in pneumatic systems, due to the limitations, the proposed designs cannot produce a complete and natural ROM of the body parts and have limitations in their presentation. Also, in some designs, due to problems such as tight fit, heavy load on bones and joints, limitation of work range, slack of wear and slippage, difficulty in dressing and undressing, in these cases, a chloroethene frame is used, which of course outer FRP jackets can also be used to reduce their weight [36]. An example of such slippage and wear on the outer jacket is shown in [42].

\subsubsection{Hydraulic Actuator}

Hydraulic pressure actuators whose fluid is oil are capable of generating large forces. To prevent fluid leakage and keep oil under pressure, their systems are complex and their commercial actuators are heavy. Therefore, specially designed hydraulic actuators have been used in rehabilitation systems. In this study, two systems were identified using hydraulic actuators. Both systems were non-standard and used specially designed actuators. Reasons to avoid using industrial hydraulic actuators include fluid leakage, impedance, weight, and fluid 
supply problems. Also, these systems are large and noisy [8]. Stienen et al. [51] presented one of the completed examples of Exoskeleton with the help of hydraulic actuators, in which the disk brake system was used in the robot members.

\subsubsection{Other Actuators}

To reduce the high resistance of electric motors, an elastic element can be added to the actuators in series, which leads to the development of the Series Elastic Actuators (SEA) concept [8]. In general, a SEA has low output resistance, good back-drivability, power output resolution and power control compared to the direct connection of the gearbox to electric motors, and also the most important element in the design of SEAs is the elastic element [47]. SEAs reduce user interface immobility and impedance to provide stable and accurate force control, thus increasing patient safety. The disadvantage of using an elastic element is the lower functional bandwidth [8]. Hydraulic SEAs are also used in some systems equipped with powerful hydraulic disc brakes. Electrical stimulation of the muscles of the body instead of using external stimuli can also create a simulation system called Functional electrical stimulation (FES), in which the weight of the system is greatly reduced. FES significantly reduces the weight of the device. From a therapeutic point of view, FES allows patients to improve muscles, improve a large part of muscle strength and power, and prevent muscle atrophy. FES, which is performed with conventional physiotherapy, has also been shown to enhance the outcome of rehabilitation. One of the disadvantages of this method is that it can cause involuntary contraction of strong muscles and cause pain in the patient. In addition, movement control using FES is difficult due to the nonlinear nature of the contracted muscles, muscle fatigue, and the dependence of contraction resulting from the quality of contact between the actuating electrodes and body tissue [8]. Also, Park et al. [66], used cam structure and a rubber band to create the required force, which has reduced the muscle fatigue of the system users in a passive actuating mode. In other design, Sanchez et al., used elastic bands to generate the force required for the actuators, although this mechanism is designed in a remote monitoring system and as a passive system [37]. Gaponov et al., "presented an example of a Twisted String Actuator (TSA), which are actuators that do not require the use of gears between motors and threads and are useful in terms of weight and cost. One of their disadvantages is that due to their dimensions, they need a lot of space to operate and it is not possible to use them for systems with a higher degree of freedom and portability" [16].

\subsection{Types of Sensors}

The importance of wearable robots is visible to all due to their wide range of applications in the fields of rehabilitation, military, medicine, increasing power and industry. In recent years, due to the increasing number of elderly and injured people in various fields who have mobility disabilities, the trend of using these robots has also increased. The sensors used in the systems also vary depending on the designs and actuators used in the systems. For example, in systems that have used pneumatic actuators, pressure sensors have been used that can measure the amount of compressed air [34, 54, 65] or in systems that have used electric motors, position, force and torque sensors have been used $[31,33,48]$ to include basic information for sending to the system control unit. One of the most common sensors used in various systems is surface electromyography (sEMG) signals of human muscles that are used as receivers of input information to control robotic systems [52]. Table 1 presents the sensors used separately for each design, which includes a pressure sensor, accelerometer, angular encoder, EMG signals [40], 6 axis Force and Torque Sensorx [88], Inertial measurement unit (IMU), bend [37], force [88] and torque sensor and position sensor, but one type to a combination of some types are used in different designs.

\subsection{Types of Power Transmission Systems}

According to the existing designs reviewed in Table 1, the transmission systems used in the designs can be classified into three main groups, which depending on the designs, one and sometimes several groups have been used in the designs: Linkage mechanism, Cable drive and Gear drive.

\subsubsection{Linkage Mechanism}

In most designs, aluminum trunks are used and the actuators are located near the desired member and the power transmission from one member to another is done through linkage [32, 48]. In other words, for example, where electric motors are used, the motor is embedded in the desired location and from both sides transmits power between the two members through the linkage connected to it, which a schematic of them is shown in $[31,60]$. The advantage of this system is that the actuators are located at the desired point and there is no need for power transmission systems from a distance farther from the desired member to the place of force effect. One of the disadvantages of these systems is the increase in member inertia due to weight gain [68].

\subsubsection{Cable-Driven Mechanism}

In some designs, it is preferred to use a cable system designed to reduce the weight of the system and transferring the actuators to a point away from the effect site. In other words, the reduction of the load caused by the device can occur by using the tendon driven mechanism system. Because the auxiliary 
force is transmitted through the tendon, the actuators can be located in any part of the body, which ultimately reduces the size of the device and reduces barriers to movement [66]. The cable-driven mechanism allows the system to be quieter and have smooth transmissions and high accuracy that are required for wearable skeletons [22]. In some designs, first, the human movement model is analyzed based on human anatomy and sports biomechanics, then the muscles are modelled as stress lines and human movement settings are obtained. Finally, the soft bionic robot is built based on the stress line model. According to the principles of anatomy and biomechanics, the muscles movement system can be simplified as a stress line model, and according to the muscle state, a muscle tension line can move from a fixed to a moving point [21]. Due to the ability to place all motors in the fixed base of the system, these mechanisms have a high power to weight ratio, which ultimately reduces the mass, size and inertia characteristics of the robot and reduces the torque output need of the motors [27].

The cable systems used for Exoskeleton and Exosuit are different. For example, in Exoskeleton systems, the path of cables and their holder is installed on the linkages, and a rigid wearable device through its rigid connection structure, which causes the limb rotation, applies the normal force to the target limb. In this case, each exoskeleton joint needs a low friction bearing system that provides rigidity against all forces and non-axial moments. In Exosuit systems, however, the actuators are fixed at a point away from the point of effect, and only the cables are routed to the point of effect through the cable system. One of the important points of the cable system design is that for complete control of $n$ joints, at least $n+1$ cable is necessary and it is necessary to have positive stress in all cables at all times to prevent slack of cables [27]. Also note that cable transfer always adds undesirable vibrations and can become loose during operation, so all aspects must be fully considered in the design. Various mechanisms have been used to move the shoulder and elbow. Kim et al. [68], used Bowden cables to activate the elbow because the point of force is away from the actuator, and a pulley mechanism is designed to activate the shoulder instead of Bowden cables to minimize energy loss.

Human skeleton produces rigid support on its own. Although the extended tendon-axis system may seem less rigid in terms of accuracy and rigidity than conventional rigid exoskeletons, it imposes fewer restrictions on arm movement and is lighter and more compact. Typically, the arm placement speed in selective rehabilitation procedures is relatively low and safe for the wearer, which gives the assistant enough time to deal with cable problems [16]. The pulley settings can be used in reducing the speed in cable transmission because in the motor, the required torque is low while the angular velocity is high, while in the joint, the torque is high and the angular velocity is low [44]. In Exosuits, in contrast, our device exerts a force on its tendon of the target limb, which applies both normal and shear forces. In designs, shear force should be minimized because it is useless in limb rotation. In these systems, this is the only pressure on the joints, which of course causes the device to be rubbed on the skin, which can also be painful. For example, to reduce the shear force, Park et al. [66], "used an activation and deactivation system consisting of a non-circular cam structure and used a rubber band as a power supply". In some systems, the device is equipped with cable anchor locks that are easily adjustable [16]. Also, in some designs, reducing the tendon diameter has led to saving the size of all mechanical parts of the transmission system (pulleys, axles, etc.) [43]. One of the reasons for the use of cable-driven systems is that their main power is the ability to carry large loads over long distances without the inherent backlash or friction in the gears. In $[44,69,73]$ shows examples of cable transmission systems.

\subsubsection{Gear-Driven Mechanism}

In some systems, such as the designs presented by Chen et al., and Xiao et al., gear transmission systems have been used [22, 63]. One of the problems of these systems is that the weight of the wearable robot has increased and also these systems have been abandoned in the study and modelling phase and no sample has been made and tested to date with the available knowledge. Of course, in cable and other systems, smaller samples of gears have been used to decrease or increase of gear ratio, the purpose of this is to change the ratio created from the motor to the final point of effect. Also, Gopura et al. [49], used a gear mechanism to create the forearm movement due to the rotation of the forearm, which has been due to creating an alignment between the rotating system and the forearm limb. In general, systems that have used the gear family generally have not shown general acceptance and practical application. Cable transmissions are also more efficient than gear transmissions, thus ensuring a better degree of system back drivability [43].

\subsection{Types of Control Units}

After studying the biomechanics of upper limb of the existing designs used, the types of actuating systems and power transmission systems, the next challenge that should be considered in the design of rehabilitation and assistant systems is the system control unit. Control systems allow the patient to follow the recorded paths accurately and approach the defined goal of the system. The control input of the devices can be different signals. For example, the forces and torques applied to the various connections of devices are known as Dynamic signals. Orientations, speeds and accelerations and positions of different parts of the device are known by kinematic signals and start signals of specific activity with Trigger signal [8]. 
The use of two dynamic and kinematic input signals or a combination of them is used in most complex strategies. The need for control and safety when assisting patients with shoulder, elbow and wrist movements plays an essential role in clinical treatment [60] and of course, in addition to patient safety, the safety of the therapist must also be considered. There are three types of rehabilitation depending on the patient-robot interaction. In the first case, the robot moves the patient's arm in a planned direction according to the defined goals, and in this case, the patient puts his arm in a relaxed position, which is called passive rehabilitation. In the second case, the patient moves his hand towards the target and the robot creates a force in that direction, which is called active-assisted position. Finally, in the third case, the robot applies the opposite force to move the patient's force, which is called active-constrained [73], which of course, a more comprehensive explanation of their control logics will be presented following.

\subsubsection{Control Strategies}

According to Maciejasz et al. [8], the breakdown of control strategies for rehabilitation and assistive robots can be classified as follows: High-level control that includes haptic stimulation, challenge-based control, coaching control, and assistive control and low-level control.

High-level control algorithms are designed to stimulate movement flexibility, While low-level position control strategies control acceptance factors, force or impedance control high-level strategies [8]. There are many high-level control strategies for teaching robotic movement. For example, Pirondini et al., in their design called ALEx, have used highlevel control algorithm with three different methods: passive, assistive and assisted-when-needed [89].

The device provides assistance to the patient to perform a specific movement, which of course is a high-level control strategy. An assistive control strategy does tasks easier and safer and causes more repetition. There is a total of four types of assistive control strategies: counterbalance-based, impedance-based, adaptive performance-based and EMG-based control.

Impedance-based control, in general, impedance-based control is used when the control of the force and position of a robotic manipulator are of concern [90]. This is common in applications that involve interaction with human where the movement speed of the robotic arm along a predefined path determines the required force that should be applied by the human. In rehabilitation and assistive devices, the robot is continuously controlling the position and the force of the patient arm to follow a specific path and the robot does not intervene until the patient deviates from the path. Deviation tolerance is considered for the permissible deviation, and if it goes out of the tolerance range, the device produces a recovery force which increases with the distance from the specified path. This predefined path is commonly chosen based on the required rehabilitation exercise the patient needs to follow. For example, Carignan et al. showed that since the torques related to the shoulder axes cannot be measured directly, an impedance controller can be used to achieve the resistance characteristics [31]. A typical observation of impedancecontrolled systems is that the selection of the controller gains could affect the performance of the whole system in terms of its stability. Since the characteristics of the human joints or limbs can differ considerably between people with common roles, the rehabilitation robot controller parameters should be changed in accordance with the existing circumstances. To tackle the issue, a recovery management system based on Artificial Neural Networks (ANN) for the upper limb is developed to choose the optimum controller gains by estimating the human arm characteristics online [91]. In [92], a variable impedance control technique is developed to control the interaction between the ankle rehabilitation device and the human's foot. It was found that changing the robot impedance in proportion to the ankle compliance enhances the performance when compared to applying constant impedance control.

Counterbalance-based control, against the movement of the limb, a weight balance of active or passive type is used to create the necessary force for movement, which increases the patient's effort by reducing gravity, and the exercises become easier [8].

EMG-based control is one of the most widely used types of control strategies in assistive technologies. This method uses sEMG signals to control or assist the patient. sEMG signals directly reflect user intents. Hence, a robot can use the user's EMG signals as input signals to the robot controller to effectively help the user move according to the user intents. However, EMG-based control is not easily possible because: (I) the role of each muscle for a particular movement varies according to joint angles, (II) a muscle is not only related to a movement but also involves other types of movement, (III) antagonist muscle activity affects joint torque, (IV) the level of activity of some muscles, such as the bi-articular muscles, is affected by the movement of other joints, (V) obtaining the same EMG signals for the same movement even with the same person is difficult, (VI) the level of activity of each muscle and its use for a particular movement varies from person to person, (VII) it is not easy to predict movement in real-time because many muscles are involved in a joint movement [46]. Humidity, human mood, ambient temperature and electrode location can affect the frequency and amplitude of the signal. The electrode should be located in the midline of the abdomen of the muscle and along the muscle fibers so that it can sense the maximum signal amplitude. It is also very important to choose the right threshold, because in the signal analysis if the starting point is too large, we have lost useful 
information, and also the starting point is disrupted by noise, and this choice is therefore very important [40].

Even if the EMG signals contain very important information, predicting shoulder movement from EMG signals in a short time is not an easy task because many muscles are involved. To overcome this problem, a fuzzy-neuro controller that can adapt to the physiological conditions of each human being online has been proposed to control the skeletal robot in some designs that the physiological control of the robot can be realized with this control method [28] and also the intelligent interface is realized using neural network.

EMG signals are usually composed of a wide range of frequencies, so it is difficult to reduce noise by filtering it. In addition, direct use of raw EMG data as input to the controller is difficult. Therefore, features must be extracted from raw EMG data. Among the various feature extraction methods, for example mean absolute value, average rectified value, mean absolute value slope, root mean square (RMS), zero crossing, waveform length or slope sign changes, most of which choose RMS values for raw EMG signal processing; because the RMS value is a measure of signal strength and is widely used in most applications [46]. Also, EMG-based fuzzy-neuro control method based on EMG has been shown to be one of the most effective control methods for controlling exoskeleton robots in previous studies. However, if the number of degrees of freedom of the exoskeleton robot increases, the control rules become more complex [52]. For example, Oujamaa et al., "have used sEMG signals from the healthy limb of the other party to control the movements of the patient" [93].

With the help of Performance-based adaptive control strategy, aspects of help such as force, path and time can be monitored in the current performance and their compatibility with the patient's performance during a certain number of previous activities can be checked [8].

In contrast to the assistive-based control strategies, the challenge-based algorithm control is based on resisting or challenging the patient's willing of movement. It is categorized into three groups: resistive, amplifying error and constraint-induced, and is a high-level strategy. In resistive strategy, the control algorithm resists the desired movements and increases the patient's effort and attention to achieve a certain task. The control techniques are based on the concept that the larger the error, the faster the progress in the recovery process. Therefore, this strategy based on increasing the observed visual error between the main path embedded and the path travelled and enhances the visual representation on the screen. Finally, in the constraint-induced strategy, the control algorithm promotes the use of the infected limb by restricting the other not infected limb similar to conventional constraintinduced therapy [8].

The haptic stimulation control algorithm is a high-level control strategy in which a robotic device is used as a tactile interface to perform activities in a virtual reality environment. Haptic simulation strategies use haptic devices and provide a sense of touch to interact with virtual reality objects $[94,95]$.

Coaching control algorithm is a non-contact strategy, which is a high-level control strategy, the system does not have physical contact with the patient and instead a monitoring system is provided to instruct the patient in his movements. Although the contactless approaches are beyond the positive solution discussed here, some of such techniques could be combined with contact approaches to enhance the feedback process [96].

Low-level control algorithm is a type of algorithms, strategy execution with proper position control, admittance, force or impedance can be used to develop a high-level rehabilitation strategy. In other words, the type of signal used as the control input is partly determined by the low-level control strategy and vice versa. The robot must also have low friction and negligible backlash to achieve satisfactory patientcooperative control strategies, which are based on impedance and admittance architectures. In addition, motor and gear units must be reversible [23].

Most exoskeleton systems use the Proportional-IntegralDerivative (PID) control approach, meaning that dynamic models of the system, as well as the upper human limb, are ignored [7, 11, 39] and the Proportional Derivative (PD) control method is used in some wearable robots [31, 41, 43] to evaluate the mechanical performance of the robot [13]. Because the human arm movement is nonlinear in nature, conventional linear control approaches have limitations when dealing with an upper limb robot. Thus, the idea of nonlinear control for upper extremity exoskeleton robots motivates a number of nonlinear control strategies, e.g., admittance controller [33], fuzzy-neuro controller [28], sliding mode control method [73], positioning controller method [57], iterative learning control scheme [45], computed torque control [7], adaptive control [60] and vision-based control method [65]. For example, to further improve safety and fault tolerance in the presence of variance of large unknown parameters or even actuator faults, Kang et al., considered adaptive controller according to the information provided by an adaptive observer without additional sensors, which of course was updated online [60]. There are basically two main types of controllers that are applied to accessories. The first group of controllers are position controllers. This type of design is used in cases where the angle of each joint must be precisely controlled. The second category of controllers is based on force/torque control. These controllers are commonly used as low-level controllers [73].

\subsubsection{Feedback to the User}

Various types of feedback may be available to the user, including visual [97], tactile [98], audio [97] and electrical 
stimulation [59]. Many systems in exoskeletons follow a similar design approach: using different control and sensing schemes, rigid kinematic chains are activated to mobilize a human-connected wearer [62]. In other words, the detection of the user's intent is done depending on the scenarios and the user's remaining capabilities and of course in different ways. For example, Pedrocchi et al. embedded systems alternatively in the main system that can be used intermittently: an EMG amplifier and a USB button (Scenario 1), an eye-tracking system (Scenario 2) and a Brain-computer interface (BCI) (Scenario 3). For example, Johnson et al., have used a joystick or a physiotherapist always observes the exercises of holding the dead man switch in his hand. Releasing the switch cuts off the engine power and immediately stops the robot [35]. This can also be done by pressing the emergency stop button [23]. Kiguchi et al. [55], used ultrasonic sensors to determine whether the user's hand was moving toward an object in the environment or not. Lam et al., used a vibrational stimulation and muscle tendons to support their contraction [98]. Oguntosin et al. [65], used visual feedback in their design to identify objects that are targeted by the upper extremities in daily activities.

A significant number of training systems are also presented in training in Virtual Reality (VR) scenarios. VR offers a very interesting patient training compared to the conventional conditions in medical units. VR can also be a unique environment in which treatment can be provided in a highly functional and motivational context and can be easily graded and recorded [43]. Since the entertainment industry has recently introduced many new devices to record the movement of healthy people to interact with VR-based games, it is expected that some of these devices will soon be adapted for rehabilitation purposes. A graphical representation offers different educational scenarios to the patient. The scenario is different from the selected training mode. These include passive mobilization, active game therapy and active ADL training. In passive mobilization, the patient's limb is moved by a robot in a previously recorded path. The purpose of this treatment is to prevent secondary complications, increase blood circulation and reduce joint and muscle stiffness [99]. In some systems, contact-less movement detection methods have been used. In these systems, reflectors are connected to the selected muscles and using motion recording systems, they finally offer the desired data to control and calculate the actual force of the muscles $[44,74,100]$. Finally, in some devices, limbs are equipped with several Radio-frequency identification (RFID) tags so that they can be detected automatically [59].

\subsection{Status and Details of Clinical Trials}

The principles of neuroplasticity suggest that these networks can be rewired through repetitive training [45]. Intense and repetitive physical rehabilitation has been shown to be useful in overcoming upper extremity deficiencies, but this treatment is intensive and expensive and its quantitative and objective assessment is difficult [34]. Table 1 provides the required information separately for each of the designs, on what kind of and how many people, the designed system has been tested clinically or in the laboratory, and with this scale, the validity of the submitted designs can be understood. In addition, it seems that the results of using devices that are currently in clinical practice have not been as positive as predicted, and more comprehensive studies on clinical evaluation have been conducted in previously published literature $[8,9,15]$.

Some previous studies have provided a specific classification for clinical trials that included them in categories 0 to Category III/IV $[8,15]$ but the number of patients and target groups and the overall type of plan have sufficed in this study. Category 0 refers to initial feasibility studies that trials performed with a small number of healthy volunteers, often using a prototype of a device, to assess its safety and clinical feasibility. Category I states pilot consideration-of-concept studies that examine clinical trials aimed at device safety testing, clinical feasibility, and potential benefit, and are performed on a small number of people with the disease. There is also no control group in the test session, or healthy individuals are used as the control group. Category II states developmentof-concept studies and reviews clinical studies to confirm the effectiveness of the device, including a standard description of the intervention, a control group, randomization and blinded outcome assessment. Finally, Category III/IV offers demonstration-of-concept studies/ proof-of-concept studies and provides more evaluation of the device's effectiveness. However, similar to the second category, these are usually multi-axis studies with a large number of participants. Clinically, the purpose of a clinical study may differ from the validity of a particular device. For therapists, a robotic device is a tool that offers a treatment protocol instead of a final product, so they are more interested in answering questions about optimal training intensity and disorders that what kind of training might be useful, whether it is robotic therapy or it should replace or complement other forms of treatment [8].

The verification classification of the proposed designs is presented separately in Fig. 8. As stated in the chart, more than $40 \%$ of the designs have been tested on healthy people and only $2 \%$ of them are finalized and commercialized designs.

\section{Challenges and Future Directions}

Due to the increasing population of the elderly and the disabled people on the one hand and the lack of therapists on the other hand, the need for robotic systems that people can easily use at home is very high. One of the biggest challenges of this 


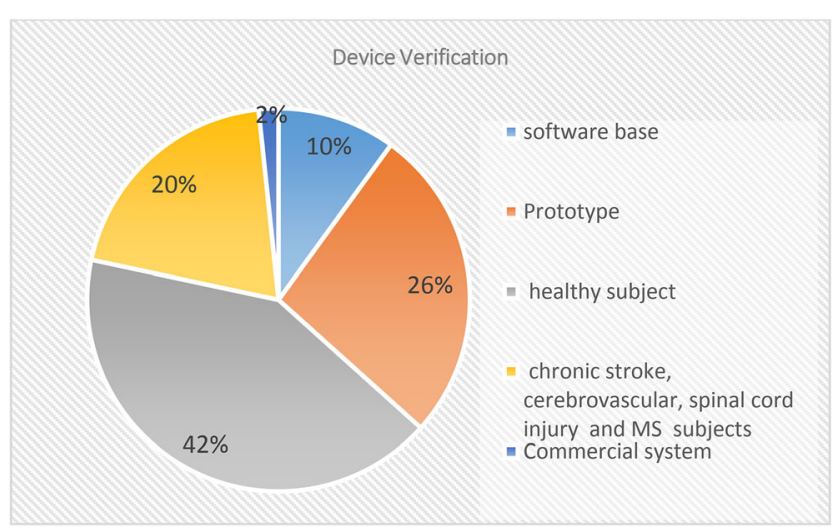

Fig. 8 Verification classification of the proposed designs

path can be reducing the price of products for the use of a wider group of these systems. The next challenge is to provide portable and intelligent systems that provide the required forces and torques depending on the user's needs. Many efforts have been made in this direction and many plans have been presented, but there are still future paths for the development of plans.One of the future direstions could be working on lighter materials in the construction of rehabilitation robotic systems. Another could be the focus on converting stationary systems to portable systems. Because it can help people do their ADLs easier than before at home. Also, in the field of soft robotic systems, the proposed designs often have a lower number of DOFs than the systems presented in rigid robotics, and one of the future paths could be to focus on producing soft robotic systems with greater DOFs. New solutions can also be developed to overcome shear forces, as well as finding new solutions to prevent slippage and wear in soft robotic systems.

\begin{abstract}
Abbreviation ADLs, Activities of Daily Living; CR, Center of Rotation; HH, humerus head; ROM, Range of Motion; HRI, Humanrobot interaction; GUI, Graphical User Interface; NMES, Neuromuscular Electrical Stimulation; NA, Not available; RTP, Repetitive task practice; VR, virtual reality; PAM, Pneumatic Artificial Muscle; SEA, Series Elastic Actuators; CGH, center of glenohumeral joint -; BCI, Brain-computer interface; FRP, Fiber-reinforced plastic; sEMG, surface Electromyogram; FES, Functional electrical stimulation; DOF, Degree of freedom; IMU, Inertial measurement unit; RMS, root mean square; PID, Proportional-Integral-Derivative; PD, Proportional Derivative; RFID, Radio-frequency identification
\end{abstract}

Code Availability Not applicable.

Authors' Contributions Conceptualization: SNM, HMFV/ Data curation: HMFV/ Analysis: HMFV/ Funding acquisition: SNM/ Investigation: HMFV, ZS/ Project administration: HMFV, SNM/ Supervision: SNM, $\mathrm{SD} /$ Writing - original draft: HMFV, ZS/ Writing - review \& editing: HMFV, ZS, HEH.

Funding This work is supported by grant EP/R026092 (FAIR-SPACE Hub) through UKRI under the Industry Strategic Challenge Fund (ISCF) for Robotics and AI Hubs in Extreme and Hazardous Environments.
Data Availability Not applicable.

\section{Declarations}

Conflict of Interest The authors declare no conflict of interest.

Ethics Approval Not applicable.

Consent to Participate Not applicable.

Consent for Publication Not applicable.

Open Access This article is licensed under a Creative Commons Attribution 4.0 International License, which permits use, sharing, adaptation, distribution and reproduction in any medium or format, as long as you give appropriate credit to the original author(s) and the source, provide a link to the Creative Commons licence, and indicate if changes were made. The images or other third party material in this article are included in the article's Creative Commons licence, unless indicated otherwise in a credit line to the material. If material is not included in the article's Creative Commons licence and your intended use is not permitted by statutory regulation or exceeds the permitted use, you will need to obtain permission directly from the copyright holder. To view a copy of this licence, visit http://creativecommons.org/licenses/by/4.0/.

\section{References}

1. O’Neill, C.T., Phipps, N.S., Cappello, L., Paganoni, S., Walsh, C.J.: A soft wearable robot for the shoulder: design, characterization, and preliminary testing. IEEE Int. Conf. Rehabil. Robot. 02129, 1672-1678 (2017). https://doi.org/ 10.1109/ICORR.2017.8009488

2. "Overview Stroke," NHS. https://www.nhs.uk/conditions/stroke/ (accessed May 30, 2020)

3. "The top 10 causes of death," 2018. https://www.who.int/newsroom/fact-sheets/detail/the-top-10-causes-of-death (accessed May 30, 2020)

4. "WHO | The Atlas of Heart Disease and Stroke." https://www. who.int/cardiovascular_diseases/resources/atlas/en/ (accessed May 30, 2020)

5. Ren, Y., Park, H., Zhang, L.: Developing a whole-arm exoskeleton robot with hand opening and closing mechanism for up - per limb stroke rehabilitation, IEEE International Conference on Rehabilitation Robotics (ICORR), pp. 761-765. Kyoto, Japan (2009). https://doi.org/10.1109/ICORR.2009.5209482

6. Zeiaee, A., Soltani-Zarrin, R., Langari, R., Tafreshi, R.: Design and kinematic analysis of a novel upper limb exoskeleton for rehabilitation of stroke patients. IEEE Int. Conf. Rehabil. Robot. 2017, 759-764 (2017). https://doi.org/10.1109/ICORR.2017. 8009339

7. Rahman, M.H., Rahman, M.J., Cristobal, O.L., Saad, M., Kenné, J.P., Archambault, P.S.: Development of a whole arm wearable robotic exoskeleton for rehabilitation and to assist upper limb movements. Robotica. 33(1), 19-39 (2015). https://doi.org/10.1017/S0263574714000034

8. Maciejasz, P., Eschweiler, J., Gerlach-Hahn, K., Jansen-Troy, A., and Leonhardt, L.: A survey on robotic devices for upper limb rehabilitation, J. Neuroeng. Rehabil., vol. 11, no. 3, (2014), doi: https://doi.org/10.1007/s00115-003-1549-7

9. Lo, A.C.: Clinical designs of recent robot rehabilitation trials. Am. J. Phys. Med. Rehabil. 91(11) SUPPL.3, 204-216 (2012). https:// doi.org/10.1097/PHM.0b013e31826bcfa3 
10. Natividad, R. F. and Yeow, C. H.” Development of a soft robotic shoulder assistive device for shoulder abduction, Proc. IEEE RAS EMBS Int. Conf. Biomed. Robot. Biomechatronics, pp. 989-993, (2016), doi: https://doi.org/10.1109/BIOROB.2016.7523758

11. Tsagarakis, N.G., Caldwell, D.G.: Development and control of a 'soft-actuated' exoskeleton for use in physiotherapy and training. Auton. Robot. 15(1), 21-33 (2003). https://doi.org/10.1023/A: 1024484615192

12. Vertechy, R., Frisoli, A., Dettori, A., Solazzi, M., and Bergamasco, M., "Development of a new exoskeleton for upper limb rehabilitation," IEEE Int. Conf. Rehabil. Robot. ICORR, pp. 188-193, (2009), doi: https://doi.org/10.1109/ICORR.2009. 5209502

13. Gopura, R. A. R. C. and Kiguchi, K.: Mechanical designs of active upper-limb exoskeleton robots state-of-the-art and design difficulties, IEEE Int. Conf. Rehabil. Robot. ICORR, pp. 178-187, (2009), doi: https://doi.org/10.1109/ICORR.2009.5209630

14. Bogue, R.: Exoskeletons and robotic prosthetics: a review of recent developments. Ind. Robot. 36(5), 421-427 (2009). https:// doi.org/10.1108/01439910910980141

15. Varghese, R.J., Freer, D., Deligianni, F., Liu, J., Yang, G.-Z.: Wearable robotics for upper-limb rehabilitation and assistance: a review on the state-of-the-art, challenges and future research. In: Tong, R. (ed.) Wearable technology in medicine and health care, pp. 23-69. Elsevier (Academic Press) (2018). https://doi.org/10. 1016/B978-0-12-811810-8.00003-8

16. Gaponov, I., Popov, D., Lee, S.J., Ryu, J.H.: Auxilio: a portable cable-driven exosuit for upper extremity assistance. Int. J. Control. Autom. Syst. 15(1), 73-84 (2017). https://doi.org/10.1007/ s12555-016-0487-7

17. "Scopus - Analyze search results," Scopus, 2020. https://wwwscopus-com.salford.idm.oclc.org/term/analyzer.uri?sid= 44ee6ed7f71b4aa2628c43b23addf774\&origin=resultslist\&src $=$ $\mathrm{s} \& \mathrm{~s}=\% 28 \mathrm{TITLE}-\mathrm{ABS}-\mathrm{KEY} \% 28$ exoskeleton+\%29+AND+ TITLE-ABS-KEY\%28robotic\%29\%29\&sort=plf-f\&sdt=b\&sot= b\&sl $=56 \&$ count $=5520 \&$ analyzeResults $=$ Analy $($ accessed Sep . 07, 2020)

18. "Scopus - Analyze search results," Scopus, 2020. https://wwwscopus-com.salford.idm.oclc.org/term/analyzer.uri?sid= 44ee6ed7f71b4aa2628c43b23addf774\&origin=resultslist\&src= $\mathrm{s} \& \mathrm{~s}=\% 28 \mathrm{TITLE}-\mathrm{ABS}-\mathrm{KEY} \% 28$ exosuit $\% 29+\mathrm{AND}+\mathrm{TITLE}-$ ABS-KEY\%28robotic\%29\%29\&sort=plf-f\&sdt=b\&sot=b\&sl= $51 \&$ count $=103 \&$ analyzeResults $=$ Analyze + res $($ accessed Sep. 07, 2020)

19. Drake, R., Vogl, A.W., Mitchell, A.W.M.: Gray's anatomy for students. Churchill Livingstone, Philadelphia (2009)

20. Pang, Z., Wang, T., Wang, Z., Yu, J., Sun, Z., and Liu, S.: Design and analysis of a wearable upper limb rehabilitation robot with characteristics of tension mechanism, Appl. Sci., vol. 10, no. 6, (2020), doi: https://doi.org/10.3390/app10062101

21. Li, N. et al.: Bio-inspired Upper Limb Soft Exoskeleton to Reduce Stroke-induced Complications, Biochem. J., pp. 1-14, (2010), doi: https://doi.org/10.1088/1748-3190/aad8d4

22. Xiao, F., Gao, Y., Wang, Y., Zhu, Y., Zhao, J.: Design of a wearable cable-driven upper limb exoskeleton based on epicyclic gear trains structure. Technol. Health Care. 25(S1), S3-S11 (2017). https://doi.org/10.3233/THC-171300

23. Nef, T., Guidali, M., Riener, R.: ARMin III - arm therapy exoskeleton with an ergonomic shoulder actuation. Appl. Bionics Biomech. 6(2), 127-142 (2009). https://doi.org/10.1080/ 11762320902840179

24. Schiele, A., Van Der Helm, F.C.T.: Kinematic design to improve ergonomics in human machine interaction. IEEE Trans. Neural Syst. Rehabil. Eng. 14(4), 456-469 (2006). https://doi.org/10. 1109/TNSRE.2006.881565
25. Koo, D., Chang, P. H., Sohn, M. K., and Shin, J. H.: Shoulder mechanism design of an exoskeleton robot for stroke patient rehabilitation, In IEEE International Conference on Rehabilitation Robotics, (2011), pp. 1-6, doi: https://doi.org/10.1109/ICORR.2011.5975505

26. Ergin, M., Patoglu, V.: ASSISTON-SE: A self-aligning shoulderelbow exoskeleton. In: IEEE International Conference on Robotics and Automation, pp. 2479-2485, Saint Paul, MN, USA (2012). https://doi.org/10.1109/ICRA.2012.6225117

27. Ball, S. J., Brown, I. E., and Scott, S. H.: MEDARM: A rehabilitation robot with 5DOF at the shoulder complex," In IEEE/ASME International Conference on Advanced Intelligent Mechatronics, AIM, (2007), pp. 1-6, doi: https://doi.org/10. 1109/AIM.2007.4412446

28. Kiguchi, K., Iwami, K., Yasuda, M., Watanabe, K., Fukuda, T.: An exoskeletal robot for human shoulder joint motion assist. IEEE/ASME Trans. Mechatronics. 8(1), 125-135 (2003). https:// doi.org/10.1109/TMECH.2003.809168

29. Kenhub, "Upper extremity: Anatomy study course |," Kenhub. https://www.kenhub.com/en/start/upper-extremity (accessed Jun. 07, 2020)

30. "Anatomy of the Forearm - Muscles and Tendons," YouTube. https://www.youtube.com/watch?v=zSjB3vEnAJ8 (accessed Jul. 11, 2020)

31. Carignan, C., Liszka, M., and Roderick, S.: Design of an arm exoskeleton with scapula motion for shoulder rehabilitation, Int. Conf. Adv. Robot. ICAR '05, Proc., pp. 524-531, (2005), doi: https://doi.org/10.1109/ICAR.2005.1507459

32. Mihelj, M., Nef, T., and Riener, R.: ARMin II - 7 DoF rehabilitation robot: Mechanics and kinematics, in Proceedings - IEEE International Conference on Robotics and Automation, (2007), no. April, pp. 4120-4125, doi: https://doi.org/10.1109/ROBOT. 2007.364112

33. Carignan, C., Tang, J., Roderick, S., Naylor, M.: A configurationspace approach to controlling a rehabilitation arm exoskeleton. In: IEEE 10th International Conference on Rehabilitation Robotics, pp. 179-187. Noordwijk, Netherlands (2007). https://doi.org/10. 1109/ICORR.2007.4428425

34. Sugar, T.G., Jiping He, Koeneman, E.J., Koeneman, J.B., Herman, R., Huang, H., Schultz, R.S., Herring, D.E., Wanberg, J., Balasubramanian, S., Swenson, P., Ward, J.A.: Design and control of RUPERT: a device for robotic upper extremity repetitive therapy. IEEE Trans. Neural Syst. Rehabil. Eng. 15(3), 336-346 (2007). https://doi.org/10.1109/TNSRE.2007.903903

35. Johnson, G.R., Carus, D.A., Parrini, G., Scattareggia Marchese, S., Valeggi, R.: The design of a five-degree-of-freedom powered orthosis for the upper limb. Proc. Inst. Mech. Eng. Part H J. Eng. Med. 215(3), 275-284 (2001). https://doi.org/10.1243/0954411011535867

36. Kobayashi, H., Ishida Y., and Suzuki, H.: Realization of all motion for the upper limb by muscle suit, In In RO-MAN 2004. 13th IEEE International Workshop on Robot and Human Interactive Communication (IEEE Catalog No. 04TH8759), (2004), pp. 631-636, doi: https://doi.org/10.1109/texcra.2004.1424995

37. Sanchez, R., et al.: Monitoring functional arm movement for home-based therapy after stroke. Annu. Int. Conf. IEEE Eng. Med. Biol. - Proc. 26 VII, 4787-4790 (2004). https://doi.org/10. 1109/iembs.2004.1404325

38. Toth, A., Fazekas, G., Arz, G., Jurak, M., and Horvath, M.: Passive robotic movement therapy of the spastic hemiparetic arm with REHAROB: Report of the first clinical test and the follow-up system improvement, In IEEE 9th International Conference on Rehabilitation Robotics, (2005), pp. 127-130, doi: https://doi.org/10.1109/ICORR.2005.1501067

39. Nef, T. and Riener, R.: ARMin - Design of a novel arm rehabilitation robot, In IEEE 9th International Conference on Rehabilitation Robotics, (2005), pp. 57-60, doi: https://doi.org/ 10.1109/ICORR.2005.1501051 
40. Li, Q., Wang, D., Du, Z., Song, Y., and Sun, L.: sEMG based control for 5 DOF upper limb rehabilitation robot system, IEEE Int. Conf. Robot. Biomimetics, ROBIO, pp. 1305-1310, (2006), doi: https://doi.org/10.1109/ROBIO.2006.340117

41. Wolbrecht, E. T., Leavitt, J., Reinkensmeyer, D. J., and Bobrow, J. E.: Control of a pneumatic orthosis for upper extremity stroke rehabilitation, In Annual International Conference of the IEEE Engineering in Medicine and Biology - Proceedings, (2006), pp. 2687-2693, doi: https://doi.org/10.1109/IEMBS.2006.259941

42. Kobayashi, H. and Nozaki, H.: Development of muscle suit for supporting manual worker, In IEEE International Conference on Intelligent Robots and Systems, (2007), pp. 1769-1774, doi: https://doi.org/10.1109/IROS.2007.4399412

43. Frisoli, A. et al:: Arm rehabilitation with a robotic exoskeleleton in virtual reality, 2007 IEEE 10th Int. Conf. Rehabil. Robot. ICORR'07, vol. 00, no. c, pp. 631-642, (2007), doi: https://doi. org/10.1109/ICORR.2007.4428491

44. Perry, J.C., Rosen, J., Burns, S.: Upper-limb powered exoskeleton design. IEEE/ASME Trans. Mechatronics. 12(4), 408-417 (2007). https://doi.org/10.1109/TMECH.2007.901934

45. Balasubramanian, S. et al:: Rupert: an exoskeleton robot for assisting rehabilitation of arm functions, Virtual Rehabil. IWVR, pp. 163-167, (2008), doi: https://doi.org/10.1109/ICVR.2008.4625154

46. Kiguchi, K., Rahman, M.H., Sasaki, M., Teramoto, K.: Development of a 3DOF mobile exoskeleton robot for human upper-limb motion assist. Robot. Auton. Syst. 56(8), 678-691 (2008). https://doi.org/10.1016/j.robot.2007.11.007

47. Stienen, A. H. A., Hekman, E. E. G., Ter Braak, H., Aalsma, A. M. M., Van Der Helm, F. C. T., and Van Der Kooij, H.: Design of a rotational hydro-elastic actuator for an active upper-extremity rehabilitation exoskeleton, In International Conference on Biomedical Robotics and Biomechatronics, (2008), pp. 881888, doi: https://doi.org/10.1109/BIOROB.2008.4762873

48. Moubarak, S., Pham, M.T., Pajdla, T., Redarce, T.: Design results of an upper extremity exoskeleton. IFMBE Proc. 22, 1687-1690 (2008). https://doi.org/10.1007/978-3-540-89208-3_401

49. Gopura, R. A. R. C. and Kiguchi, K.: Development of a 6 DOF exoskeleton robot for human upper-limb motion assist, Proc. 2008 4th Int. Conf. Inf. Autom. Sustain. ICIAFS 2008, pp. 13-18, (2008), doi: https://doi.org/10.1109/ICIAFS.2008.4783986

50. Garrec, P., Friconneau, J.P., Méasson, Y., Perrot, Y.: ABLE, an innovative transparent exoskeleton for the upper-limb. In: IEEE/ RSJ International Conference on Intelligent Robots and Systems, pp. 1483-1488, Nice, France (2008). https://doi.org/10.1109/ IROS.2008.4651012

51. Stienen, A.H.A., Hekman, E.E.G., Prange, G.B., Jannink, M.J.A., Aalsma, A.M.M., van der Helm, F.C.T., van der Kooij, H.: Dampace: design of an exoskeleton for force-coordination training in upper-extremity rehabilitation. J. Med. Devices, Trans. ASME. 3(3), 1-10 (2009). https://doi.org/10.1115/1.3191727

52. Gopura, R.A.R.C., Kiguchi, K., Yi, Y.: SUEFUL-7: A 7DOF upper-limb exoskeleton robot with muscle-model-oriented EMG-based control. 2009 IEEE/RSJ Int. Conf. Intell. Robot. Syst. IROS. 2009, 1126-1131 (2009). https://doi.org/10.1109/ IROS.2009.5353935

53. Umemura, A., Saito, Y., and Fujisaki, K.; A study on powerassisted rehabilitation robot arms operated by patient with upper limb disabilities, In IEEE International Conference on Rehabilitation Robotics, ICORR, (2009), pp. 451-456, doi: https://doi.org/10.1109/ICORR.2009.5209512

54. Klein, J., Spencer, S., Allington, J., Bobrow, J.E., Reinkensmeyer, D.J.: Optimization of a parallel shoulder mechanism to achieve a high-force, low-mass, robotic-arm exoskeleton. IEEE Trans. Robot. 26(4), 710-715 (2010). https://doi.org/10.1109/TRO.2010.2052170

55. Kiguchi, K., Kose, Y., Hayashi, Y.: Task-oriented perception-assist for an upper-limb powerassist exoskeleton robot. In: Proc. World
Automation Congress (WAC), pp. 1-6. Kobe, Japan (2010) http:// ieexplore.ieee.org/xpls/abs_all.jsp?arnumber $=5665314$

56. Dehez, B. and Sapin, J.: ShouldeRO, an Alignment-Free TwoDOF Rehabilitation Robot for the Shoulder Complex, IEEE International Conference on Rehabilitation Robotics, (2011), doi: https://doi.org/10.1109/ICORR.2011.5975339

57. Galiana, I., Hammond, F. L., Howe, R. D., and Popovic, M. B.: Wearable soft robotic device for post-stroke shoulder rehabilitation: Identifying misalignments, In IEEE International Conference on Intelligent Robots and Systems, (2012), pp. 317-322, doi: https://doi.org/10.1109/IROS.2012.6385786

58. Pignolo, L., Dolce, G., Basta, G., Lucca, L. F., Serra, S., and Sannita, W. G.: Upper limb rehabilitation after stroke: ARAMIS a robomechatronic innovative approach and prototype, In Proceedings of the IEEE RAS and EMBS International Conference on Biomedical Robotics and Biomechatronics, (2012), pp. 1410-1414, doi: https:// doi.org/10.1109/BioRob.2012.6290868

59. Pedrocchi, A., Ferrante, S., Ambrosini, E., Gandolla, M., Casellato, C., Schauer, T., Klauer, C., Pascual, J., Vidaurre, C., Gföhler, M., Reichenfelser, W., Karner, J., Micera, S., Crema, A., Molteni, F., Rossini, M., Palumbo, G., Guanziroli, E., Jedlitschka, A., Hack, M., Bulgheroni, M., d'Amico, E., Schenk, P., Zwicker, S., Duschau-Wicke, A., Miseikis, J., Graber, L., Ferrigno, G.: MUNDUS project: MUltimodal Neuroprosthesis for daily upper limb support. J. Neuroeng. Rehabil. 10, 1 (2013). https://doi.org/ 10.1186/1743-0003-10-66

60. Kang, H.B., Wang, J.H.: Adaptive control of 5 DOF upper-limb exoskeleton robot with improved safety. ISA Trans. 52(6), 844 852 (2013). https://doi.org/10.1016/j.isatra.2013.05.003

61. Ren, Y., Kang, S.H., Park, H.S., Wu, Y.N., Zhang, L.Q.: Developing a multi-joint upper limb exoskeleton robot for diagnosis, therapy, and outcome evaluation in neurorehabilitation. IEEE Trans. Neural Syst. Rehabil. Eng. 21(3), 490-499 (2013). https://doi.org/10.1109/TNSRE.2012.2225073

62. Xu, K., Qiu, D.: Experimental design verification of a compliant shoulder exoskeleton. In: IEEE International Conference on Robotics and Automation, pp. 3894-3901. Karlsruhe, Germany (2013). https://doi.org/10.1109/ICRA.2013.6631125

63. Chen, Y., Li, G., Zhu, Y., Zhao, J., Cai, H.: Design of a 6-DOF upper limb rehabilitation exoskeleton with parallel actuated joints. Biomed. Mater. Eng. 24(6), 2527-2535 (2014). https://doi.org/10. 3233/BME-141067

64. Chonnaparamutt, W., Supsi, W.: SEFRE: Semiexoskeleton Rehabilitation System. Appl. Bionics Biomech. 2016, 1-12 (2016). https://doi.org/10.1155/2016/8306765

65. Oguntosin, V.W., Mori, Y., Kim, H., Nasuto, S.J., Kawamura, S., Hayashi, Y.: Design and Validation of exoskeleton actuated by soft modules toward neurorehabilitation-vision-based control for precise reaching motion of upper limb. Front. Neurosci. 11(JUL), 1-20 (2017). https://doi.org/10.3389/fnins.2017.00352

66. Park, D., Cho, K.: Development and evaluation of a soft wear-able weight support device for reducing muscle fatigue on shoulder. PLOS One. (2017). https://doi.org/10.1371/journal.pone.0173730

67. Natividad R.F., Hong S.W., Miller-Jackson T.M., Yeow CH. (2019) The Exosleeve: a soft robotic exoskeleton for assisting in activities of daily living. In: Carrozza M., Micera S., Pons J. (eds) Wearable robotics: challenges and trends. WeRob 2018. Biosystems \& Biorobotics, vol 22. Springer, Cham. https://doi. org/10.1007/978-3-030-01887-0_78

68. Kim, Y.G., Xiloyannis, M., Accoto, D., Masia, L.: Development of a Soft Exosuit for Industrial Applications. In: 7th IEEE International Conference on Biomedical Robotics and Biomechatronics (Biorob), pp. 324-329. Enschede, Netherlands (2018). https://doi.org/10.1109/BIOROB.2018.8487907

69. Lessard, S., Pansodtee, P., Robbins, A., Trombadore, J.M., Kurniawan, S., Teodorescu, M.: A soft exosuit for flexible 
upper-extremity rehabilitation. IEEE Trans. Neural Syst. Rehabil. Eng. 26(8), 1604-1617 (2018). https://doi.org/10.1109/TNSRE. 2018.2854219

70. Tiseni, L., et al.: On the edge between soft and rigid : an assistive shoulder exoskeleton with hyper-redundant kinematics. In: IEEE 16th International Conference on Rehabilitation Robotics (ICORR), pp. 618-624. ON, Canada, Toronto (2019). https:// doi.org/10.1109/ICORR.2019.8779546

71. Carolina, A., Mendes, D. A., Kutilek, P., Hejda, J., Smrcka, P., and Havlas, V.: Design of Smart Orthosis of Upper Limb for Rehabilitation, In World Congress on Medical Physics and Biomedical Engineering, (2019), pp. 773-778, doi: https://doi. org/10.1007/978-981-10-9038-7

72. Varghese, R.J., Lo, B.P.L., Yang, G.Z.: Design and prototyping of a bio-inspired kinematic sensing suit for the shoulder joint: precursor to a multi-DoF shoulder exosuit. IEEE Robot. Autom. Lett. 5(2), 540 547 (2020). https://doi.org/10.1109/LRA.2019.2963636

73. Pont, D., et al.: ExoFlex: an upper-limb cable-driven Exosuit. In: Silva, M., Luís Lima, J., Reis, L., Sanfeliu, A., Tardioli, D. (eds.) Robot 2019: Fourth Iberian Robotics Conference. ROBOT 2019. Advances in Intelligent Systems and Computing, vol 1093. Springer, Cham (2020). https://doi.org/10.1007/978-3-03036150-1 34

74. Varghese, R. J., Nguyen, A., Burdet, E., Yang, G.-Z., and Lo, B. P. L.: Nonlinearity Compensation in a Multi-DoF Shoulder Sensing Exosuit for Real-Time Teleoperation, (2020), [Online]. Available: http://arxiv.org/abs/2002.09195

75. Chang, J.J., Tung, W.L., Wu, W.L., Huang, M.H., Su, F.C.: Effects of robot-aided bilateral force-induced isokinetic arm training combined with conventional rehabilitation on arm motor function in patients with chronic stroke. Arch. Phys. Med. Rehabil. 88(10), 1332-1338 (2007). https://doi.org/10.1016/j.apmr.2007. 07.016

76. Piña-Martínez, E., Roberts, R., Leal-Merlo, S., and RodriguezLeal, E.: Vision System-Based Design and Assessment of a Novel Shoulder Joint Mechanism for an Enhanced Workspace Upper Limb Exoskeleton, Appl. Bionics Biomech., (2018), doi: https://doi.org/10.1155/2018/6019381

77. Micera, S., Carrozza, M.C., Guglielmelli, E., Cappiello, G., Zaccone, F., Freschi, C., Colombo, R., Mazzone, A., Delconte, C., Pisano, F., Minuco, G., Dario, P.: A simple robotic system for neurorehabilitation. Auton. Robot. 19(3), 271-284 (2005). https:// doi.org/10.1007/s10514-005-4749-0

78. Culmer, P.R., Jackson, A.E., Makower, S.G., Cozens, J.A., Levesley, M.C., Mon-Williams, M., Bhakta, B.: A novel robotic system for quantifying arm kinematics and kinetics: description and evaluation in therapist-assisted passive arm movements poststroke. J. Neurosci. Methods. 197(2), 259-269 (2011). https://doi. org/10.1016/j.jneumeth.2011.03.004

79. Galinski, D., Sapin, J., and Dehez, B.: Optimal Design of an Alignment-Free Two-DOF Rehabilitation Robot for the Shoulder Complex, IEEE Int. Conf. Rehabil. Robot., (2013), doi: https://doi.org/10.1109/ICORR.2013.6650502

80. Mayr, A., Kofler, M., Saltuari, L.: ARMOR: Elektromechanischer roboter für das bewegungstraining der oberen extremität nach schlaganfall. Prospektive randomisierte kontrollierte pilotstudie. Handchir. Mikrochir. Plast. Chir. 40(1), 66-73 (2008). https:// doi.org/10.1055/s-2007-989425

81. Jarrassé, N., et al.: Robotic exoskeletons: A perspective for the rehabilitation of arm coordination in stroke patients. Front. Hum. Neurosci. 8(DEC), 1-13 (2014). https://doi.org/10.3389/fnhum. 2014.00947

82. "ShoulderX," suitX. https://www.suitx.com/shoulderx (accessed Jul. 31, 2020)

83. "EksoWorks," Ekso Bionics. https://eksobionics.com/eksoworks/ (accessed Jul. 31, 2020)
84. "Myomo -," Medical Robotics Solutions for Stroke, BPI, Upper Limb Paralysis. https://myomo.com/ (accessed Jul. 31, 2020)

85. "Paexo Shoulder -," Ottobock Industrials. https://paexo.com/ paexo-shoulder/?lang=en (accessed Jul. 31, 2020)

86. "EksoUE," Ekso Bionics. https://eksobionics.com/eksohealth/ eksoue/ (accessed Jul. 31, 2020)

87. Morales, R., Badesa, F.J., García-Aracil, N., Sabater, J.M., PérezVidal, C.: Pneumatic robotic systems for upper limb rehabilitation. Med. Biol. Eng. Comput. 49(10), 1145-1156 (2011). https://doi. org/10.1007/s11517-011-0814-3

88. "Large Force Sensing Resistor," FlexiForce A401 Sensor Tekscan. https://www.tekscan.com/products-solutions/forcesensors/a401 (accessed Oct. 12, 2020)

89. Pirondini, E., Coscia, M., Marcheschi, S., Roas, G., Salsedo, F., Frisoli, A., Bergamasco, M., Micera, S.: Evaluation of the effects of the arm light exoskeleton on movement execution and muscle activities: a pilot study on healthy subjects. J. Neuroeng. Rehabil. 13(1), 1-21 (2016). https://doi.org/10.1186/s12984-016-0117-x

90. Hogan, N.: Impedance control: an approach to manipulation. In: 1984 American Control Conference, pp. 304-313, San Diego, CA, USA (1984). https://doi.org/10.23919/ACC.1984.4788393

91. Erol, D., Mallapragada, V., and Sarkar, N.: Adaptable force control in robotic rehabilitation, In Proceedings - IEEE International Workshop on Robot and Human Interactive Communication, (2005), pp. 649-654, doi: https://doi.org/10.1109/ROMAN. 2005.1513853

92. Tsoi, Y. H. and Xie, S. Q.: Impedance control of ankle rehabilitation robot, 2008 IEEE Int. Conf. Robot. Biomimetics, ROBIO 2008, pp. 840-845, (2009), doi: https://doi.org/10.1109/ROBIO. 2009.4913109

93. Oujamaa, L., Relave, I., Froger, J., Mottet, D., Pelissier, J.Y.: Rehabilitation of arm function after stroke. Literature review. Ann. Phys. Rehabil. Med. 52(3), 269-293 (2009). https://doi. org/10.1016/j.rehab.2008.10.003

94. Casadio, M., Sanguineti, V., Morasso, P.G., Arrichiello, V.: Braccio di Ferro: a new haptic workstation for neuromotor rehabilitation. Technol. Health Care. 14(3), 123-142 (2006). https:// doi.org/10.3233/thc-2006-14301

95. Gupta, A., O'Malley, M.K., Patoglu, V., Burgar, C.: Design, control and performance of RiceWrist: a force feedback wrist exoskeleton for rehabilitation and training. Int. J. Robot. Res. 27(2), 233 251 (2008). https://doi.org/10.1177/0278364907084261

96. Ramirez, A.V., Kurita, Y.: A Soft Exoskeleton Jacket with Pneumatic Gel Muscles for Human Motion Interaction. International Conference on Human-Computer Interaction. 11573, 587-603 (2019). https://doi. org/10.1007/978-3-030-23563-5

97. Krebs, H.I., Hogan, N., Aisen, M.L., Volpe, B.T.: Robot-aided Neurorehabilitation. IEEE Trans. Rehabil. Eng. 6(1), 75-87 (1998)

98. Lam, P., Hebert, D., Boger, J., Lacheray, H., Gardner, D., Apkarian, J., Mihailidis, A.: A haptic-robotic platform for upperlimb reaching stroke therapy: preliminary design and evaluation results. J. Neuroeng. Rehabil. 5, 1-13 (2008). https://doi.org/10. 1186/1743-0003-5-15

99. Nef, T., Guidali, M., Klamroth-Marganska, V., and Riener, R.: ARMin - Exoskeleton Robot for Stroke Rehabilitation, pp. 127130, (2009), doi: https://doi.org/10.1007/978-3-642-03889-1 35

100. Zhang, Q., Liu, R., Chen, W., Xiong, C.: Simultaneous and continuous estimation of shoulder and elbow kinematics from surface EMG signals. Front. Neurosci. 11(MAY), 1-12 (2017). https:// doi.org/10.3389/fnins.2017.00280

Publisher's Note Springer Nature remains neutral with regard to jurisdictional claims in published maps and institutional affiliations. 
Hamed Majidi Fard Vatan graduated in mechanical engineering from the University of Tehran, Iran, in 2012. He worked as a mechanical engineer, project manager and R\&D manager and he collaborated in various largescale projects with international teams such as Persian, Turkish, Chinese, Indians, and Europeans in automotive, manufacturing, data center, steel making, and industrial compressors. Since 2020, Hamed started his $\mathrm{PhD}$ in robotics at the University of Salford, Manchester. He is working on rehabilitation and augmentation robotics devices for patients and astronauts. His research interests include robotics, manufacturing, soft robotics, biomechanics, bioinspired robot systems, robotics in healthcare and rehabilitation, machine learning and deep learning.

Samia Nefti-Meziani is director of the centre for Autonomous Systems \& Advanced Robotics at University of Salford. She is a cofounder and executive board member of the UK's National Robotics Network, former vice chairman of IEEE RAS UK and a member of the UKGovernment Robotics Growth Partnership. She has a proven strategic leader of multinational, multi-sector, multi-faceted and complex robotics \& artificial intelligence research programmes. She has worked very closely with the space, aerospace, nuclear, automotive and food sectors to deliver proof of concept and innovative low-cost robotics solutions. She has 25 years' experience in advanced research in the areas of embodied intelligence and advanced robotics.

Steve Davis obtained his $\mathrm{PhD}$ in Advanced Robotics from the University of Salford in 2005 and became a Team Leader at the Italian Institute of Technology in 2008, he is currently Chair in Advanced Robotics at the University of Salford (UK). His research interests include manufacturing, lightweight advanced actuators and artificial muscles, 'soft' robotics, human robot interaction, dexterous robot hands, biomimetics and biologically inspired robot systems and robotics in healthcare and rehabilitation. He has published extensively on 'soft' robotics, biomimetics, grippers and humanoid technologies as well as automation. He has guest edited journals and been on the programme committee for many IEEE conferences including and has attracted significant research funding both nationally and at a European level.

Zahra Saffari received the B.Sc. and M.Sc. degree in Electrical and Electronic Engineering from Hakim Sabzevari University and Shahid Rajaee University, Tehran, Iran in 2009 and 2013, respectively. She has more than ten years of teaching experience in different electrical, electronics and computer courses in Ministry of Education and universities in Iran. She is currently researching on machine learning and robotics in greater Manchester, United Kingdom. Her research interests include machine learning, deep learning, robotics, autonomous systems, IoT and wireless sensor networks.

Haitham El-Husseiny received the B.Sc. degree in electronics and communication engineering from the faculty of engineering (Shoubra), Benha University, Egypt, in 2007, and the M.Sc. and Ph.D. degrees in mechatronics and robotics engineering from the Egypt-Japan University of Science and Technology (E-JUST), Alexandria, Egypt, in 2013 and 2016, respectively. He was working as an assistant professor of robotics engineering with the electrical engineering department, faculty of engineering (Shoubra), Benha University and currently he is on sabbatical leave. Since August 2019, Haitham has been working as a senior research fellow in soft robotics with the University of Salford, Manchester, UK. His research interests include soft robots, soft haptics, teleoperation, model predictive control, and applied intelligence. 